COMMUNICATIONS IN

ANALYSIS AND GEOMETRY

Volume 12, Number 5, 967-1007, 2004

\title{
Geometry of Yang-Baxter Maps: pencils of conics and quadrirational mappings
}

\begin{abstract}
V. E. Adler ${ }^{1,2}$, A. I. Bobenko ${ }^{1,3}$, And Yu. B. Suris ${ }^{1,4}$
Birational Yang-Baxter maps ('set-theoretical solutions of the Yang-Baxter equation') are considered. A birational map $(x, y) \mapsto$ $(u, v)$ is called quadrirational, if its graph is also a graph of a birational map $(x, v) \mapsto(u, y)$. We obtain a classification of quadrirational maps on $\mathbb{C P}^{1} \times \mathbb{C P}^{1}$, and show that all of them satisfy the Yang-Baxter equation. These maps possess a nice geometric interpretation in terms of linear pencil of conics, the Yang-Baxter property being interpreted as a new incidence theorem of the projective geometry of conics.
\end{abstract}

Keywords: Yang-Baxter map, Yang-Baxter equation, set-theoretical solution, 3D-consistency, quadrirational map

\section{Introduction.}

Our major object of interest in this paper are Yang-Baxter maps, i.e. invertible maps satisfying the Yang-Baxter equation

$$
R_{23} \circ R_{13} \circ R_{12}=R_{12} \circ R_{13} \circ R_{23} .
$$

Here $R_{i j}: \mathcal{X}^{3} \rightarrow \mathcal{X}^{3}$ acts on the $i$-th and $j$-th factors of the cartesian product $\mathcal{X}^{3}$ and is identical on the rest one. The maps $R_{i j}$, considered as mappings

\footnotetext{
${ }^{1}$ Institut für Mathematik, Technische Universität Berlin, Str. des 17. Juni 136, 10623 Berlin, Germany.

${ }^{2}$ On leave from: Landau Institute for Theoretical Physics, Institutsky pr. 12, 142432 Chernogolovka, Russia. E-mail: adler@itp.ac.ru. Supported by the Alexander von Humboldt Stiftung and by the RFBR grant 02-01-00144.

${ }^{3}$ E-mail: bobenko@math.tu-berlin.de. Partially supported by the SFB 288 "Differential Geometry and Quantum Physics" and the DFG Research Center "Mathematics for Key Technologies" (FZT 86) in Berlin.

${ }^{4}$ E-mail: suris@sfb288. math.tu-berlin. de. Supported by the SFB 288 "Differential Geometry and Quantum Physics".
} 
$\mathcal{X} \times \mathcal{X} \rightarrow \mathcal{X} \times \mathcal{X}$, may not coincide with each other, for example they can correspond to different values of some parameters.

This notion was introduced in [5] under the name of set-theoretical solutions of the Yang-Baxter equation; a number of examples for the case when $\mathcal{X}$ is a finite set was found in [9], and classification results for this case were achieved in [7], see also [11]. It turned out that the following non-degeneracy notion is natural and useful for such maps. Let

$$
R:(x, y) \mapsto(u, v)=(u(x, y), v(x, y))
$$

this map is called non-degenerate if for any fixed $x \in \mathcal{X}$, the map $v(x, \cdot)$ : $\mathcal{X} \mapsto \mathcal{X}$ is bijective, and for any fixed $y \in \mathcal{X}$, the map $u(\cdot, y): \mathcal{X} \mapsto \mathcal{X}$ is bijective.

Important examples of Yang-Baxter maps were found in $[1,12,10,6$, $15,8]$. It was pointed out in [6] that the natural ambient category for these examples is not that of sets, but that of irreducible algebraic varieties, so that the role of morphisms is played by birational isomorphisms rather than by bijections. So, in this setting it is supposed that $R$ is a birational isomorphism of $\mathcal{X} \times \mathcal{X}$ to itself. Then the rational maps $v(x, \cdot): \mathcal{X} \mapsto \mathcal{X}$ and $u(\cdot, y): \mathcal{X} \mapsto \mathcal{X}$ are well defined for generic $x$, resp. $y$, and $R$ is called non-degenerate, if both these maps are birational isomorphisms of $\mathcal{X}$ to itself.

In [15] the term "Yang-Baxter maps" was proposed instead of "settheoretical solutions", and various notions of integrability were studied. In particular, commuting monodromy maps were constructed and Lax representations were discussed. A general construction of Lax representations was given subsequently in [14]. In the present paper we want to support the change of terminology proposed in [15], since we consider the term "Yang-Baxter maps" much more suitable and adequate. Correspondingly, we will say birational Yang-Baxter maps instead of "rational set-theoretical $R$-matrix" (which was proposed initially in [6]). Moreover, since the term "non-degenerate" is heavily overloaded in the mathematical literature, we propose a new term for non-degenerate (in the above sense) birational YangBaxter maps, namely we will call them quadrirational. We find this notation highly suggestive, in particular because of the following way to visualize the Yang-Baxter equation (1). Assign elements of $\mathcal{X}$ ("fields") to the edges of a quadrilateral, so that the arrow from the down-left to the up-right corner of the left square on Fig. 1 encodes the map $R:(x, y) \mapsto(u, v)$. Then the opposite arrow naturally encodes the inverse map $R^{-1}:(u, v) \mapsto(x, y)$, and the quadrirationality (non-degeneracy in the previous terminology) means the existence of the rational maps $\bar{R}:(u, y) \mapsto(x, v)$ and $\bar{R}^{-1}:(x, v) \mapsto(u, y)$, 
which are encoded by two arrows on the right square on Fig. 1. We call $\bar{R}, \bar{R}^{-1}$ the companion maps for $R$.
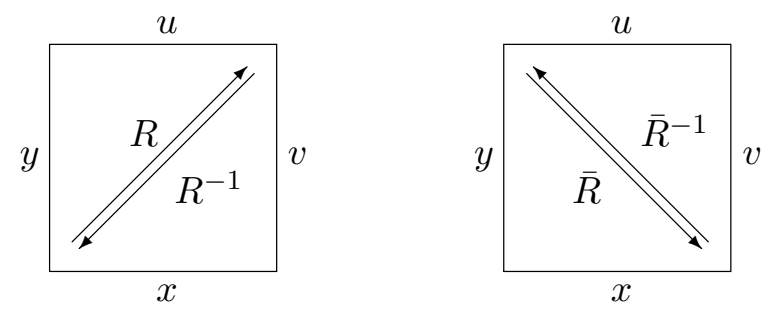

Figure 1: A map on $\mathcal{X} \times \mathcal{X}$, its inverse and its companions

The Yang-Baxter equation (1) can be depicted as consistency of the maps $R$ attached to 6 facets of a cube. The right-hand side of (1) corresponds to the chain of maps along three front faces of the cube on Fig. 2:

$$
(y, z) \stackrel{R_{23}}{\longmapsto}\left(y_{3}, z_{2}\right), \quad\left(x, z_{2}\right) \stackrel{R_{13}}{\longmapsto}\left(x_{3}, z_{12}\right), \quad\left(x_{3}, y_{3}\right) \stackrel{R_{12}}{\longmapsto}\left(x_{23}, y_{13}\right)
$$

while its left-hand side corresponds to the analogous chain along the rear faces (it is supposed that opposite faces of the cube carry the same maps). Eq. (1) assures that two ways of obtaining $\left(x_{23}, y_{13}, z_{12}\right)$ from the initial data $(x, y, z)$ lead to the same results.

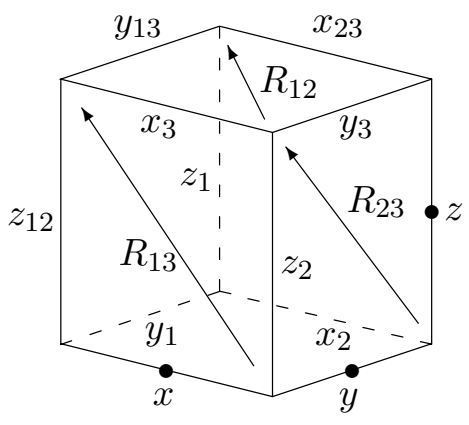

Figure 2: Right-hand side of Yang-Baxter equation

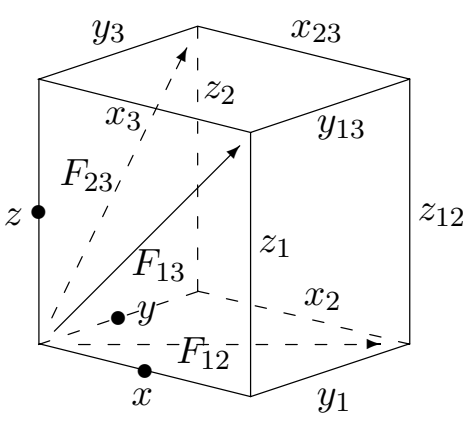

Figure 3: 3D consistency

An alternative way to express this idea is known under the name of $3 D$ consistency property (cf. [2]). It uses another setting of initial data on the edges, as shown on Fig. 3. Also, to distinguish between these two notions, 
we use a different notation for the maps $\left(F_{i j}\right.$ instead of $\left.R_{i j}\right)$ on this figure. Applying the maps to initial data one obtains 6 new values at the first step:

$$
(x, y) \stackrel{F_{12}}{\longmapsto}\left(x_{2}, y_{1}\right), \quad(x, z) \stackrel{F_{13}}{\longmapsto}\left(x_{3}, z_{1}\right), \quad(y, z) \stackrel{F_{23}}{\longmapsto}\left(y_{3}, z_{2}\right),
$$

and applying the maps once more, one gets two values for each of the fields $x_{23}, y_{13}, z_{12}$. We say that the maps $F_{i j}$ are $3 \mathrm{D}$ consistent if the two values for each of these fields coincide (for arbitrary initial data).

Comparing the two pictures, we see that (at least in the situation when the maps are quadrirational) the two notions are equivalent, if $F_{i j}=\bar{R}_{i j}$.

As pointed out above, all known birational Yang-Baxter maps turn out to possess a more strong property of being quadrirational. In our opinion, the notion of a quadrirational map is of a great interest for its own, and our results indicate that it is intimately related with the $3 \mathrm{D}$ consistency (although the precise relation still has to be uncovered). In any case, a more detailed understanding of the nature of quadrirational maps seems to be a necessary pre-requisite for any attempt to classify 3D consistent maps (or, equivalently, Yang-Baxter maps). In the present paper we furnish this task in the case $\mathcal{X}=\mathbb{C P}^{1}$.

We start in Sect. 2 with a precise definition of quadrirationality, and then give a nice construction, related to the geometry of conics, which leads to a large class of quadrirational maps. It allows also to prove the $3 \mathrm{D}$ consistency of the maps corresponding to a triple of conics belonging to one linear pencil. (See Theorem 5; this typical incidence statement of the projective geometry might be known previously, but we did not succeed in finding it in the literature.) In other words, linear pencils of conics carry a remarkable family of novel Yang-Baxter maps. Upon a rational parametrization of all encountered conics, these Yang-Baxter maps act on $\mathbb{C P}^{1} \times \mathbb{C P}^{1}$.

Then, we proceed with the task of classifying quadrirational maps on $\mathbb{C P}^{1} \times \mathbb{C P}^{1}$. An explicit description of all such maps is given in Sect. 4 . They are $\boldsymbol{b} \boldsymbol{i}$-Möbius, i.e. have the form

$$
F: \quad u=\frac{a(y) x+b(y)}{c(y) x+d(y)}, \quad v=\frac{A(x) y+B(x)}{C(x) y+D(x)},
$$

where $a(y), \ldots, d(y)$ are polynomials in $y$, while $A(x), \ldots, D(x)$ are polynomials in $x$. It turns out that there exist three subclasses of such maps, depending on the degree of the polynomials involved; they are denoted by pair of numbers as [1:1], [1:2] and [2:2], corresponding to the highest degrees of the coefficients of both fractions in (3). The most rich and interesting subclass is [2:2]; it is analyzed in detail, while the two simpler ones are briefly discussed in Appendix 3. 
By classifying quadrirational maps on $\mathbb{C P}^{1} \times \mathbb{C P}^{1}$, it is natural to factor out the action of the group $(\mathcal{M} \ddot{o} b)^{4}$ of Möbius transformations acting independently on each field $x, y, u, v$ (recall that Möbius transformations are birational isomorphisms of $\left.\mathbb{C P}^{1}\right)$. The construction of Sect. 4 is not well suited for this purpose. This is done in Sect. 5-7 with the help of different techniques based on the analysis of the singularities of the maps, which seems to be an adequate language and admits further generalizations. We obtain the following result.

Theorem 1. Any quadrirational map on $\mathbb{C P}^{1} \times \mathbb{C P}^{1}$ of subclass [2:2] is equivalent, under some change of variables from $(\mathcal{M o ̈ b})^{4}$, to exactly one of the following five maps:

$$
\begin{aligned}
& u=\alpha y P, \quad v=\beta x P, \quad P=\frac{(1-\beta) x+\beta-\alpha+(\alpha-1) y}{\beta(1-\alpha) x+(\alpha-\beta) y x+\alpha(\beta-1) y}, \\
& u=\frac{y}{\alpha} P, \quad v=\frac{x}{\beta} P, \quad P=\frac{\alpha x-\beta y+\beta-\alpha}{x-y}, \\
& u=\frac{y}{\alpha} P, \quad v=\frac{x}{\beta} P, \quad P=\frac{\alpha x-\beta y}{x-y}, \\
& u=y P, \quad v=x P, \quad P=1+\frac{\beta-\alpha}{x-y}, \\
& u=y+P, \quad v=x+P, \quad P=\frac{\alpha-\beta}{x-y},
\end{aligned}
$$

with some suitable constants $\alpha, \beta$.

The map $\left(F_{\mathrm{V}}\right)$ appeared in [1] as the superposition formula for Bäcklund transformations of the $\mathrm{KdV}$ equation. Other four maps apparently did not appear in the literature in an explicit form, though they are related to some of the equations from the list of [2]. This paper was devoted to the classification of a different version of $3 \mathrm{D}$ consistency, where the fields are attached to the vertices rather than to the edges of the cube.

Remarkably, the five maps of Theorem 1 describe five particular cases of the geometrical construction of Sect. 2. To mention several further features of these maps: each one of them is an involution and coincides with its companion maps, so that all four arrows on Fig. 1 are described by the same formulae. Moreover, these maps have the following additional symmetry:

$$
x \leftrightarrow y, u \leftrightarrow v, \alpha \leftrightarrow \beta .
$$

Note also that these maps come with the intrisically built-in parameters $\alpha, \beta$. Neither their existence nor a concrete dependence on parameters are presupposed in Theorem 1. The geometric interpretation of these parameters 
in terms of singularities of the map will be given in Sect. 7. It will be shown that in the formulas above the parameter $\alpha$ is naturally assigned to the edges $x, u$, while $\beta$ is naturally assigned to the edges $y, v$.

The most remarkable fact about the maps $F_{\mathrm{I}}-F_{\mathrm{V}}$ is their $3 \mathrm{D}$ consistency. For any $\mathcal{T}=$ I, II, III, IV or V, denote the corresponding map $F_{\mathcal{T}}$ of Theorem 1 by $F_{\mathcal{T}}(\alpha, \beta)$, indicating the parameters explicitly.

Theorem 2. For any $\alpha_{1}, \alpha_{2}, \alpha_{3} \in \mathbb{C}$, the maps $R_{i j}=F_{\mathcal{T}}\left(\alpha_{i}, \alpha_{j}\right)$ (acting nontrivially on the $i$-th and the $j$-th factors of $\left.\left(\mathbb{C P}^{1}\right)^{3}\right)$ satisfy the YangBaxter equation (1). Analogously, the maps $F_{i j}=F_{\mathcal{T}}\left(\alpha_{i}, \alpha_{j}\right)$ are $3 D$ consistent.

Actually, the $3 \mathrm{D}$ concistency of quadrirational maps on $\mathbb{C P}^{1} \times \mathbb{C P}^{1}$ takes place not only for the normal forms $F_{\mathcal{T}}\left(\alpha_{i}, \alpha_{j}\right)$ but under much more general circumstances. The only condition for the subclass [2:2] consists in matching singularities along all edges of the cube (see Theorem 19). Similar statements hold also for other two subclasses [1:1] and [1:2], so that in the scalar case the properties of being quadrirational and of being $3 \mathrm{D}$ consistent are related very closely.

It would be intriguing to establish such a relation for more general algebraic varieties, e.g. for $\mathbb{C P}^{n} \times \mathbb{C P}^{n}$. Of course, in this case the classification of quadrirational maps has to be performed modulo automorphisms of $\mathbb{C P}^{n}$ (Cremona group) on each edge, which makes it a nearly impossible task. Nevertheless, the singularities matching approach seems promising also in studying this case.

\section{Geometry of conics and quadrirational maps.}

We start with giving a slightly more general definition of quadrirational maps. Let $\mathcal{X}_{i}(i=1,2)$ be irreducible algebraic varieties over $\mathbb{C}$. Consider a rational map $F: \mathcal{X}_{1} \times \mathcal{X}_{2} \rightarrow \mathcal{X}_{1} \times \mathcal{X}_{2}$. It will be identified with its graph an algebraic variety $\Gamma_{F} \subset \mathcal{X}_{1} \times \mathcal{X}_{2} \times \mathcal{X}_{1} \times \mathcal{X}_{2}$.

Definition 3. A map $F$ is called quadrirational, if for any fixed pair $(X, Y) \in \mathcal{X}_{1} \times \mathcal{X}_{2}$ except maybe some closed subvarieties of codimension $\geq 1$, the variety $\Gamma_{F}$ intersects each one of the sets $\{X\} \times\{Y\} \times \mathcal{X}_{1} \times \mathcal{X}_{2}$, $\mathcal{X}_{1} \times \mathcal{X}_{2} \times\{X\} \times\{Y\}, \mathcal{X}_{1} \times\{Y\} \times\{X\} \times \mathcal{X}_{2}$ and $\{X\} \times \mathcal{X}_{2} \times \mathcal{X}_{1} \times\{Y\}$ exactly once, i.e. if $\Gamma_{F}$ is a graph of four rational maps $F, F^{-1}, \bar{F}, \bar{F}^{-1}: \mathcal{X}_{1} \times \mathcal{X}_{2} \mapsto$ $\mathcal{X}_{1} \times \mathcal{X}_{2}$. 
This definition is immediately applicable to the following map. Consider a pair of nondegenerate conics $Q_{1}, Q_{2}$ on the plane $\mathbb{C P}^{2}$, so that both $Q_{i}$ are irreducible algebraic varieties isomorphic to $\mathbb{C P}^{1}$. Take $X \in Q_{1}, Y \in Q_{2}$, and let $\ell=\overline{X Y}$ be the line through $X, Y$ (well-defined if $X \neq Y$ ). Generically, the line $\ell$ intersects $Q_{1}$ at one further point $U \neq X$, and intersects $Q_{2}$ at one further point $V \neq Y$. This defines a map $\mathcal{F}:(X, Y) \mapsto(U, V)$, see Fig. 4 for the $\mathbb{R}^{2}$ picture.

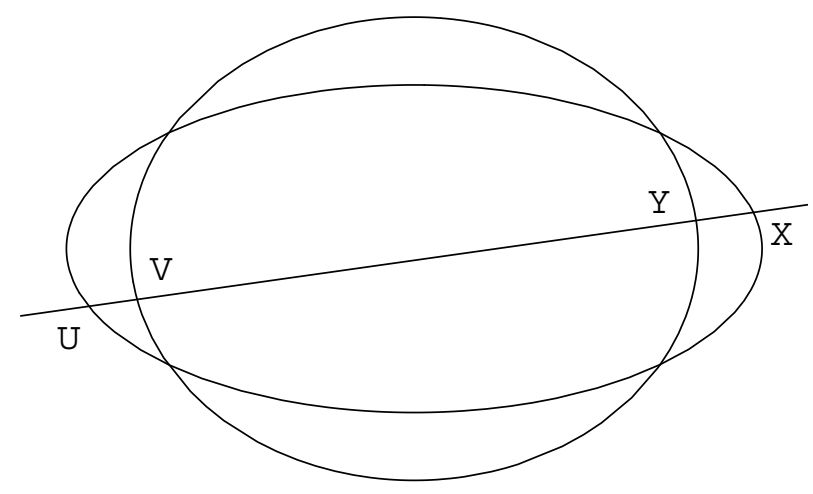

Figure 4: A quadrirational map on a pair of conics

Proposition 4. The map $\mathcal{F}: Q_{1} \times Q_{2} \mapsto Q_{1} \times Q_{2}$ is quadrirational. It is an involution and moreover coincides with its both companions. Intersection points $X \in Q_{1} \cap Q_{2}$ correspond to the singular points $(X, X)$ of the map $\mathcal{F}$.

Proof. Intersection of a conic with a line is described by a quadratic equation. Knowing one root of a quadratic equation allows us to find the second one as a rational function of the input data.

Generically, two conics intersect in four points, however, degeneracies can happen. Five possible types $\mathrm{I}-\mathrm{V}$ of intersection of two conics are described in detail in [3]:

I: four simple intersection points;

II: two simple intersection points and one point of tangency;

III: two points of tangency;

IV: one simple intersection point and one point of the second order tangency; 
$\mathrm{V}$ : one point of the third order tangency.

Using rational parametrizations of the conics:

$$
\mathbb{C P}^{1} \ni x \mapsto X(x) \in Q_{1} \subset \mathbb{C P}^{2}, \quad \text { resp. } \quad \mathbb{C P}^{1} \ni y \mapsto Y(y) \in Q_{2} \subset \mathbb{C P}^{2},
$$

it is easy to see that $\mathcal{F}$ pulls back to the map $F:(x, y) \mapsto\left(x_{2}, y_{1}\right)$ which is quadrirational on $\mathbb{C P}^{1} \times \mathbb{C P}^{1}$. One shows by a direct computation that the maps $F$ for the above five situations are exactly the five maps listed in Theorem 1.

Example of type I. We use non-homogeneous coordinates $\left(W_{1}, W_{2}\right)$ on the affine part $\mathbb{C}^{2}$ of $\mathbb{C P}^{2}$. Consider a pencil of conics through four points $O=(0,0),(0,1),(1,0),(1,1) \in \mathbb{C P}^{2}$ (any four points on $\mathbb{C P}^{2}$, no three of which lie on a straight line, can be brought into these four by a projective transformation). Conics of this pencil are described by the equation (in non-homogeneous coordinates $\left(W_{1}, W_{2}\right)$ on $\left.\mathbb{C P}^{2}\right)$ :

$$
Q(\alpha): W_{2}\left(W_{2}-1\right)=\alpha W_{1}\left(W_{1}-1\right)
$$

A rational parametrization of such a conic is given, e.g., by

$$
X(x)=\left(W_{1}(x), W_{2}(x)\right)=\left(\frac{x-\alpha}{x^{2}-\alpha}, \frac{x(x-\alpha)}{x^{2}-\alpha}\right) .
$$

Here the parameter $x$ has the interpretation of the slope of the line $\overline{O X}$. The values of $x$ for the four points of the base locus of the pencil on $Q(\alpha)$ are $x=\alpha, \infty, 0$ and 1. A straightforward computation shows that if $Q_{1}=Q(\alpha)$, $Q_{2}=Q(\beta)$ then the map $F$ defined above coincides with $\left(F_{\mathrm{I}}\right)$.

Example of type V. Consider a pencil of conics having a triple tangency point at $\left(W_{1}: W_{2}: W_{3}\right)=(0: 1: 0)$ (in homogeneous coordinates on $\left.\mathbb{C P}^{2}\right)$. Conics of this pencil and their rational parametrization are given by formulae

$$
Q(\alpha): \quad W_{2}-W_{1}^{2}-\alpha=0, \quad X(x)=\left(W_{1}(x), W_{2}(x)\right)=\left(x, x^{2}+\alpha\right) .
$$

If $Q_{1}=Q(\alpha), Q_{2}=Q(\beta)$ then the map $F$ defined above is $\left(F_{\mathrm{V}}\right)$.

The following theorem delivers a geometric interpretation of the statement of Theorem 2 . 
Theorem 5. Let $Q_{i}, i=1,2,3$ be three non-degenerate members of a linear pencil of conics. Let $X \in Q_{1}, Y \in Q_{2}$ and $Z \in Q_{3}$ be arbitrary points on these conics. Define the maps $\mathcal{F}_{i j}$ as above, corresponding to the pair of conics $\left(Q_{i}, Q_{j}\right)$. Set $\left(X_{2}, Y_{1}\right)=\mathcal{F}_{12}(X, Y),\left(X_{3}, Z_{1}\right)=\mathcal{F}_{13}(X, Z)$, and $\left(Y_{3}, Z_{2}\right)=\mathcal{F}_{23}(Y, Z)$. Then

$$
\begin{gathered}
X_{23}=\overline{X_{3} Y_{3}} \cap \overline{X_{2} Z_{2}} \in Q_{1}, \quad Y_{13}=\overline{X_{3} Y_{3}} \cap \overline{Y_{1} Z_{1}} \in Q_{2}, \\
Z_{12}=\overline{Y_{1} Z_{1}} \cap \overline{X_{2} Z_{2}} \in Q_{3} .
\end{gathered}
$$

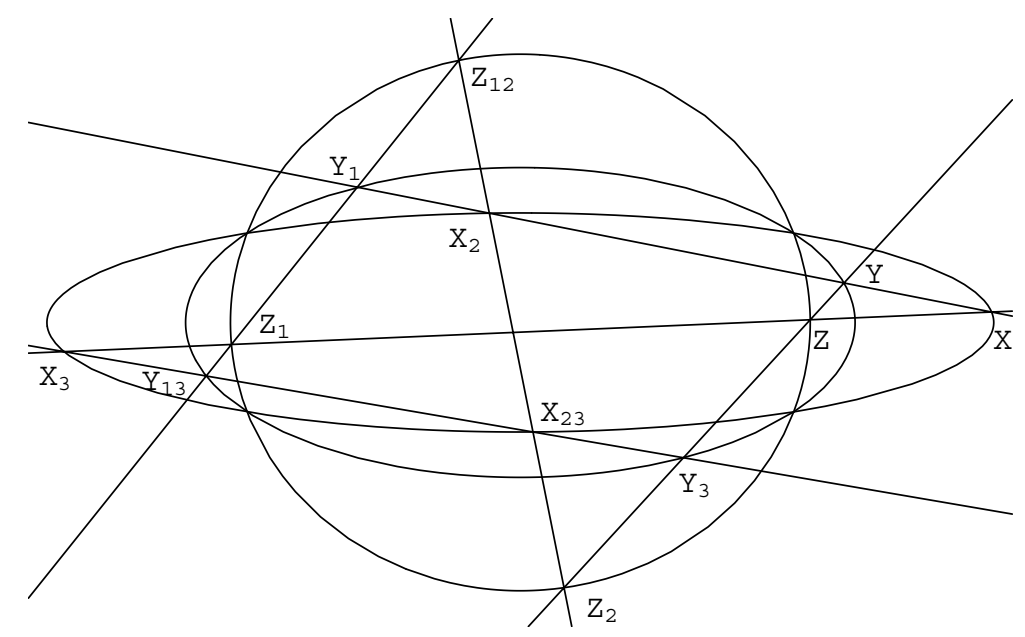

Figure 5: 3D consistency at a linear pencil of conics

Proof. We will work with equations of lines and conics on $\mathbb{C P}^{2}$ in homogeneous coordinates, and use the same notations for geometric objects and homogeneous polynomials vanishing on these objects. We start with the following data: two conics $Q_{1}$ and $Q_{2}$, three points $X \in Q_{1}, Y \in Q_{2}$, and $Z$. Construct the lines $a=\overline{Y Z}, b=\overline{X Z}$ and $c=\overline{X Y}$, respectively. Let

$$
\begin{array}{ll}
X_{2}=\left(c \cap Q_{1}\right) \backslash X, & Y_{1}=\left(c \cap Q_{2}\right) \backslash Y, \\
X_{3}=\left(b \cap Q_{1}\right) \backslash X, & Y_{3}=\left(a \cap Q_{2}\right) \backslash Y .
\end{array}
$$

Next, construct the line $C=\overline{X_{3} Y_{3}}$, and let

$$
X_{23}=\left(C \cap Q_{1}\right) \backslash X_{3}, \quad Y_{13}=\left(C \cap Q_{2}\right) \backslash Y_{3} .
$$

Finally, construct the lines $A=\overline{Y_{1} Y_{13}}$ and $B=\overline{X_{2} X_{23}}$. 
We have four points $X, X_{2}, X_{3}$ and $X_{23}$ on the conic $Q_{1}$, and two pairs of lines $(C, c)$ and $(B, b)$ through two pairs of these points each. Therefore, there exists $\mu_{1} \in \mathbb{C P}^{1}$ such that the conic $Q_{1}$ has the equation $Q_{1}=0$ with

$$
Q_{1}=\mu_{1} B b+C c .
$$

Similarly, the conic $Q_{2}$ has the equation $Q_{2}=0$ with

$$
Q_{2}=\mu_{2} A a+C c .
$$

Consider the conic

$$
Q_{1}-Q_{2}=\mu_{1} B b-\mu_{2} A a=0 .
$$

It belongs to a linear pencil of conics spanned by $Q_{1}$ and $Q_{2}$. Further, the point $Z=a \cap b$ lies on this conic. Therefore, it has to coincide with $Q_{3}$, which has therefore the equation $Q_{3}=0$ with

$$
Q_{3}=\mu_{1} B b-\mu_{2} A a .
$$

Further, the two points $Z_{2}=a \cap B$ and $Z_{1}=b \cap A$ also lie on $Q_{3}$. Since $Z_{2} \in B$, we have $B=\overline{X_{2} Z_{2}}$. Similarly, since $Z_{1} \in A$, we have $A=\overline{Y_{1} Z_{1}}$. Finally, we find that the point $Z_{12}=A \cap B=\overline{Y_{1} Z_{1}} \cap \overline{X_{2} Z_{2}}$ also lies on $Q_{3}$, which is equivalent to (5).

\section{Quadrirational and bi-Möbius maps.}

We proceed with a general study of quadrirational maps in the case $\mathcal{X}=$ $\mathbb{C P}^{1}$. In this case we can immediately make some conclusions about their nature. First of all, for a fixed value of $y$ (with a possible exception of finitely many values), the maps $F, \bar{F}$ induce a birational correspondence between $\mathbb{C P}^{1} \ni x$ and $\mathbb{C P}^{1} \ni u$. Similarly, for a fixed value of $x$ (with a possible exception of finitely many values), the maps $F, \bar{F}^{-1}$ induce a birational correspondence between $\mathbb{C P}^{1} \ni y$ and $\mathbb{C P}^{1} \ni v$. Therefore, a quadrirational map has to be bi-Möbius, i.e. be given by the formulas (3) from the Introduction. Equivalently, one can say that the variety $\Gamma_{F}$ is given by the following two (irreducible) polynomial equations:

$$
F: \phi(x, y, u)=0, \quad \Phi(y, x, v)=0,
$$

where

$$
\begin{aligned}
& \phi(x, y, u)=c(y) x u+d(y) u-a(y) x-b(y), \\
& \Phi(y, x, v)=C(x) y v+D(x) v-A(x) y-B(x) .
\end{aligned}
$$


In order for a bi-Möbius map to be quadrirational, the following nondegeneracy conditions have to be satisfied:

$$
\text { (i) } \quad u_{x} v_{y}-u_{y} v_{x} \not \equiv 0, \quad \text { (ii) } \quad u_{x} \not \equiv 0, v_{y} \not \equiv 0, \quad \text { (iii) } \quad u_{y} \not \equiv 0, \quad v_{x} \not \equiv 0 .
$$

Condition (i) is necessary for the existence of the inverse map; conditions (ii) are necessary for the existence of the companion maps and are equivalent to

$$
r(y)=a(y) d(y)-b(y) c(y) \not \equiv 0, \quad R(x)=A(x) D(x)-B(x) C(x) \not \equiv 0 ;
$$

finally, conditions (iii) assure that the map is really two-dimensional: if one of them is violated, then, making the corresponding Möbius transformations in $u$ or/and $v$, we come to the essentially one-dimensional map with $u=x$, resp. $v=y$.

The above reasoning holds for the inverse and companion maps as well, so that for a quadrirational map all four maps $F, F^{-1}, \bar{F}, \bar{F}^{-1}$ have to be bi-Möbius. So, the companion maps have to be given by

$$
\begin{array}{rlrl}
\bar{F}: & x=\frac{d(y) u-b(y)}{-c(y) u+a(y)}, & v & =\frac{\widehat{A}(u) y+\widehat{B}(u)}{\widehat{C}(u) y+\widehat{D}(u)}, \\
\bar{F}^{-1}: \quad y & =\frac{D(x) v-B(x)}{-C(x) v+A(x)}, \quad u=\frac{\widehat{a}(v) x+\widehat{b}(v)}{\widehat{c}(v) x+\widehat{d}(v)} .
\end{array}
$$

In other words, the variety $\Gamma_{F}$ can be alternatively described by either pair of polynomial equations

$$
\begin{aligned}
& \bar{F}: \quad \phi(x, y, u)=0, \quad \widehat{\Phi}(y, u, v)=0, \\
& \bar{F}^{-1}: \quad \Phi(y, x, v)=0, \quad \widehat{\phi}(x, v, u)=0,
\end{aligned}
$$

where

$$
\begin{aligned}
\widehat{\Phi}(y, u, v) & =\widehat{C}(u) y v+\widehat{D}(u) v-\widehat{A}(u) y-\widehat{B}(u), \\
\widehat{\phi}(x, v, u) & =\widehat{c}(v) x u+\widehat{d}(v) u-\widehat{a}(v) x-\widehat{b}(v) .
\end{aligned}
$$

We set

$$
\widehat{r}(v)=\widehat{a}(v) \widehat{d}(v)-\widehat{b}(v) \widehat{c}(v), \quad \widehat{R}(u)=\widehat{A}(u) \widehat{D}(u)-\widehat{B}(u) \widehat{C}(u) .
$$

Finally, the inverse map has to be given by

$$
F^{-1}: \quad x=\frac{\widehat{d}(v) u-\widehat{b}(v)}{-\widehat{c}(v) u+\widehat{a}(v)}, \quad y=\frac{\widehat{D}(u) v-\widehat{B}(u)}{-\widehat{C}(u) v+\widehat{A}(u)},
$$


so that the fourth equivalent description of the variety $\Gamma_{F}$ is given by the pair of polynomial equations

$$
F^{-1}: \quad \widehat{\phi}(x, v, u)=0, \quad \widehat{\Phi}(y, u, v)=0 .
$$

It is natural to think of the polynomials $\Phi, \phi, \widehat{\Phi}$ and $\widehat{\phi}$ as attached to the edges $x, y, u$ and $v$ of the quadrilateral on Fig. 1, respectively.

For an arbitrary bi-Möbius map both companion maps are rational. Indeed, consider, for instance, the companion map $\bar{F}:(u, y) \mapsto(x, v)$. The first equation in (3) is immediately solved with respect to $x$, and the result has to be substituted into the second equation in (3), in order to express $v$ through $u, y$. We come to the following formulas:

$$
\bar{F}: \quad x=X(y, u)=\frac{d(y) u-b(y)}{-c(y) u+a(y)}, \quad v=\frac{p(y, u)}{q(y, u)} .
$$

This is bi-Möbius, as in (7), if and only if the rational function $p(y, u) / q(y, u)$ is linear-fractional with respect to $y$. Similarly, the companion map $\bar{F}^{-1}$ : $(x, v) \mapsto(u, y)$ is a rational map of the form

$$
\bar{F}^{-1}: \quad y=Y(x, v)=\frac{D(x) v-B(x)}{-C(x) v+A(x)}, \quad u=\frac{P(x, v)}{Q(x, v)},
$$

and is bi-Möbius, as in (8), if and only if $P(x, v) / Q(x, v)$ is a linear-fractional function with respect to $x$. For the inverse map $(u, v) \mapsto(x, y)$ we have the system

$$
u=\frac{P(x, v)}{Q(x, v)}, \quad v=\frac{p(y, u)}{q(y, u)},
$$

where the first equation has to be solved for $x$, and the second one has to be solved for $y$. We come to the following conclusion: a bi-Möbius map (3) is quadrirational, if and only if its both companion maps are bi-Möbius. In this case the inverse map is also bi-Möbius.

\section{First steps toward classification.}

According to the nondegeneracy conditions (iii) above, we will suppose from now on that

$$
\operatorname{deg}_{y} \phi \geq 1, \quad \operatorname{deg}_{x} \Phi \geq 1 .
$$

Lemma 6. For a bi-Möbius map to be quadrirational it is necessary that

$$
\operatorname{deg}_{y} \phi \leq 2, \quad \operatorname{deg}_{x} \Phi \leq 2 .
$$


Proof. Eliminating $v$ between $\Phi(y, x, v)=0$ and $\widehat{\Phi}(y, u, v)=0$, we find the polynomial equation which holds on $\Gamma_{F}$ :

$$
\Phi \widehat{\Phi}_{v}-\Phi_{v} \widehat{\Phi}=0
$$

The polynomial on the left-hand side depends on $(x, y, u)$ only; it does not vanish identically (this would violate the nondegeneracy conditions). Therefore, the above equation has to be a consequence of the first equation in (3) (or in (6)). In other words, we obtain:

$$
\Phi \widehat{\Phi}_{v}-\Phi_{v} \widehat{\Phi}=\mu(x, y, u) \phi
$$

with some polynomial factor $\mu \not \equiv 0$. The left-hand side is at most quadratic in $y$. Therefore, $\operatorname{deg}_{y} \phi \leq 2$.

Lemma 7. If for a quadrirational map (3) $\operatorname{deg}_{x} \Phi=1$, then $\operatorname{deg}_{u} \widehat{\Phi}=1$. Conversely, two arbitrary polynomials $\Phi(y, x, v)$ and $\widehat{\Phi}(y, u, v)$, each linear in all three arguments, define a quadrirational map $F:(x, y) \mapsto(u, v)$.

Proof. Under the condition $\operatorname{deg}_{x} \Phi=1$, we have for the polynomials $p(y, u)$, $q(y, u)$ in (9) the following expressions:

$$
\begin{aligned}
& p(y, u)=(-c(y) u+a(y))(A(X) y+B(X)), \\
& q(y, u)=(-c(y) u+a(y))(C(X) y+D(X)),
\end{aligned}
$$

where $X=(d(y) u-b(y)) /(-c(y) u+a(y))$. They are at most linear in $u$, therefore the same holds for $\widehat{A}(u) y+\widehat{B}(u), \widehat{C}(u) y+\widehat{D}(u)$. Conversely, for any two polynomials $\Phi(y, x, v)$ and $\widehat{\Phi}(y, u, v)$ linear in all arguments, the left-hand side of (11) is linear in $x, u$ and at most quadratic in $y$. If it is irreducible, then it gives $\phi(x, y, u)$, otherwise it coincides with $\phi$ up to a factor $\mu=\mu(y)$. This shows that the map $F$ is bi-Möbius, and the proof for $\bar{F}^{ \pm 1}$ and $F^{-1}$ is analogous.

Remark 1. It can happen that on the last step in the previous proof one gets $\phi=\phi(x, u)$, i.e. the dependence on $y$ drops out, thus leading to degenerate maps $F$ with $u_{y}=0$ (excluded from consideration). This happens, for instance, if $\widehat{\Phi}(y, u, v)=\Phi(y, u, v)$, when $\phi=x-u$.

According to Lemma 7, all quadrirational maps belong to one of the 
three subclasses:

$$
\begin{aligned}
& {[1: 1] \quad \operatorname{deg}_{x} \Phi=\operatorname{deg}_{u} \widehat{\Phi}=\operatorname{deg}_{y} \phi=\operatorname{deg}_{v} \widehat{\phi}=1 ;} \\
& {[1: 2] \quad \operatorname{deg}_{x} \Phi=\operatorname{deg}_{u} \widehat{\Phi}=1, \quad \operatorname{deg}_{y} \phi=\operatorname{deg}_{v} \widehat{\phi}=2, \quad \text { or vice versa; }} \\
& {[2: 2] \quad \operatorname{deg}_{x} \Phi=\operatorname{deg}_{u} \widehat{\Phi}=\operatorname{deg}_{y} \phi=\operatorname{deg}_{v} \widehat{\phi}=2 .}
\end{aligned}
$$

Moreover, Lemma 7 gives an explicit description of a family of quadrirational maps containing all maps of subclasses [1:1] and [1:2], in terms of two polynomials attached to the opposite (rather than adjacent) edges of the quadrilateral on Fig. 1. Of course, it remains to classify all such maps modulo independent Möbius transformations on $x, y, u, v$. We return to the (simpler) classes [1:1] and [1:2] in Appendix 3, and concentrate in the main text on the most interesting subclass [2:2]. So, from now on we assume that

$$
\operatorname{deg}_{x} \Phi=2, \quad \operatorname{deg}_{y} \phi=2 .
$$

It turns out that in this case it is also possible to find an explicit description of all quadrirational maps in terms of two polynomials attached to the opposite edges.

Theorem 8. For a quadrirational map (3), let $\operatorname{deg}_{x} \Phi=\operatorname{deg}_{y} \phi=2$. Then the polynomials $\Phi(y, x, v)$ and $\widehat{\Phi}(y, u, v)$ corresponding to the opposite edges of the elementary quadrilateral are related by the formula

$$
\widehat{\Phi}(y, u, v)=(\gamma u+\delta)^{2} \Phi\left(y, \frac{\alpha u+\beta}{\gamma u+\delta}, v\right) .
$$

Conversely, for an arbitrary polynomial $\Phi(y, x, v)$, linear in $y, v$ and quadratic in $x$, define the polynomial $\widehat{\Phi}(y, u, v)$ as in (13). Then the pair of polynomials $(\Phi, \widehat{\Phi})$ determines a quadrirational map.

Proof. Under the assumption $\operatorname{deg}_{y} \phi=2$, the factor $\mu$ in the formula (11) does not depend on $y$, thus being a polynomial $\mu(x, u)$ of bidegree $(1,1)$. Moreover, it is irreducible. Indeed, suppose that $\mu\left(x_{0}, u\right) \equiv 0$. If $x_{0} \neq \infty$, then set $\Psi=\Psi(y, v)=\Phi\left(y, x_{0}, v\right)$, otherwise take $\Psi=\Psi(y, v)$ equal to the $x^{2}$-coefficient of $\Phi$. Then Eq. (11) implies $\Psi \widehat{\Phi}_{v}-\Psi_{v} \widehat{\Phi} \equiv 0$, and by further differentiation $\Psi \widehat{\Phi}_{u v}-\Psi_{v} \widehat{\Phi}_{u} \equiv 0$. Therefore, we find: $\widehat{\Phi} \widehat{\Phi}_{u v}-\widehat{\Phi}_{u} \widehat{\Phi}_{v} \equiv 0-$ a contradiction to the nondegeneracy assumption. So, $\mu$ is of the form

$$
\mu=\gamma x u+\delta x-\alpha u-\beta, \quad \alpha \delta-\beta \gamma \neq 0 .
$$


Consider the polynomial

$$
\bar{\Phi}(y, u, v)=(\gamma u+\delta)^{2} \Phi\left(y, \frac{\alpha u+\beta}{\gamma u+\delta}, v\right) .
$$

Eq. (11) implies: $\bar{\Phi} \widehat{\Phi}_{v}-\bar{\Phi}_{v} \widehat{\Phi}=0$, therefore $\widehat{\Phi}=\nu(y, u) \bar{\Phi}$, and since both $\bar{\Phi}$ and $\widehat{\Phi}$ are linear in $y$ and quadratic in $u$, we conclude that $\widehat{\Phi}=$ const $\bar{\Phi}$. This proves the first claim of the theorem.

To prove the second one, we start with an arbitrary $\Phi(y, x, v)$ and construct $\widehat{\Phi}(y, u, v)$ as in (13). Without loss of generality we can take simply $\widehat{\Phi}(y, u, v)=\Phi(y, u, v)$ (otherwise applying a Möbius transformation in $u$ ). Then the polynomial $\Phi \widehat{\Phi}_{v}-\Phi_{v} \widehat{\Phi}$ is divisible by $x-u$. In other words, there holds (11) with $\mu=x-u$, and with the quotient $\phi$ linear in $x, u$ and at most quadratic in $y$. This demonstrates that the map $F$ is bi-Möbius, and for $\bar{F}^{ \pm 1}$ and $F^{-1}$ everything is similar.

Remark 2. On the last step of the previous proof, it could well happen that $\phi$ is less then quadratic in $y$, so that the resulting map is actually in the subclass [1:2] $\left(\operatorname{deg}_{x} \Phi=\operatorname{deg}_{u} \widehat{\Phi}=2\right.$ and $\left.\operatorname{deg}_{y} \phi=\operatorname{deg}_{v} \widehat{\phi}=1\right)$.

Remark 3. A naive counting suggests that the families of quadrirational maps constructed in this section depends on 2 essential parameters. Indeed, two generic polynomials $\Phi, \widehat{\Phi}$ from the Lemma 7 contain $2 \times 8=16$ parameters, 2 are scaled out by homogeneity, and $4 \times 3=12$ ones correspond to the Möbius freedom of all variables $x, y, u, v$. Analogously, the polynomial $\Phi$ from Theorem 8 contains 12 parameters, one is scaled out, and $3 \times 3=9$ ones correspond to the Möbius freedom of $x, y, v$.

\section{Singularities.}

We proceed with the in-depth study of quadrirational maps with the property (12), which will eventually lead to their complete classification. In performing such a classification, it is natural to factor out the action by the automorphisms group of $\mathcal{X}$ on all four variables (edges) $x, y, u, v$ independently. In the present case $\mathcal{X}=\mathbb{C P}^{1}$, we factor out the action by independent Möbius transformation on all of $x, y, u, v$.

According to the conclusion of Sect. 3, we have to study the condition under which the companion maps of the given one are bi-Möbius. Recall Eqs. (9) for the companion map $\bar{F}$. Under the condition (12), the polynomials 
$p(y, u), q(y, u)$ are given by

$$
\begin{aligned}
& p(y, u)=(-c(y) u+a(y))^{2}(A(X) y+B(X)), \\
& q(y, u)=(-c(y) u+a(y))^{2}(C(X) y+D(X)) .
\end{aligned}
$$

They are of degree 5 in $y$ and of degree 2 in $u$. Similarly, in Eqs. (10) for the companion map $\bar{F}^{-1}$ the polynomials $P(x, v), Q(x, v)$ are of degree 5 in $x$ and of degree 2 in $v$.

Obviously, in order that the companion map $\bar{F}:(y, u) \mapsto(v, x)$ be biMöbius of the form (7), it is necessary and sufficient that

$$
p(y, u)=\rho(y)(\widehat{A}(u) y+\widehat{B}(u)), \quad q(y, u)=\rho(y)(\widehat{C}(u) y+\widehat{D}(u)),
$$

where $\rho(y)$ is a polynomial of degree 4 . The polynomials $\widehat{A}(u), \ldots, \widehat{D}(u)$ are quadratic. Similarly, for the companion map $\bar{F}^{-1}:(v, x) \mapsto(y, u)$ to be bi-Möbius of the form (8), it is necessary and sufficient that

$$
P(x, v)=\mathrm{P}(x)(\widehat{a}(v) x+\widehat{b}(v)), \quad Q(x, v)=\mathrm{P}(x)(\widehat{c}(v) x+\widehat{d}(v)),
$$

where $\mathrm{P}(x)$ is a polynomial of degree 4 , and $\widehat{a}(v), \ldots, \widehat{d}(v)$ are quadratic polynomials.

Next, consider the conditions (15), (16). It is enough to consider the first one, since for the second everything is similar. For (15) to hold, there have to exist 4 numbers $y_{i}, i=1, \ldots, 4$, such that $p\left(y_{i}, u\right) \equiv 0, q\left(y_{i}, u\right) \equiv 0$ (of course, the zeros $y_{i}$ have to be counted with their multiplicity).

Lemma 9. If $p(\eta, u) \equiv 0, q(\eta, u) \equiv 0$ for some $\eta \in \mathbb{C P}^{1}$, then $\eta$ is with necessity a root of $r(y)=a(y) d(y)-b(y) c(y)$.

Proof. From the formulas (14) one sees that if $a(\eta) d(\eta)-b(\eta) c(\eta) \neq 0$ then

$$
\begin{aligned}
& A\left(\frac{d u-b}{-c u+a}\right) \eta+B\left(\frac{d u-b}{-c u+a}\right) \equiv 0, \\
& C\left(\frac{d u-b}{-c u+a}\right) \eta+D\left(\frac{d u-b}{-c u+a}\right) \equiv 0,
\end{aligned}
$$

where $a=a(\eta), \ldots, d=d(\eta)$. These two formulas, being identities in $u$, are equivalent to $A(x) \eta+B(x) \equiv 0, C(x) \eta+D(x) \equiv 0$, which, in turn, means that the second component of the map (3) is degenerate $(R(x) \equiv 0)$ - a contradiction. Hence, $a(\eta) d(\eta)-b(\eta) c(\eta)=0$. Lemma is proved. 
Proposition 10 (cancellation of simple zero). In order for $\eta$ to be a common zero of $p(y, u)$ and $q(y, u)$ (considered as polynomials in $y$ ), i.e.

$$
p(\eta, u)=q(\eta, u)=0,
$$

it is necessary and sufficient that the point $(\xi, \eta)$ defined by the system

$$
a(\eta) \xi+b(\eta)=0, \quad c(\eta) \xi+d(\eta)=0,
$$

satisfies also the system

$$
A(\xi) \eta+B(\xi)=0, \quad C(\xi) \eta+D(\xi)=0 .
$$

In particular, $\xi$ is a zero of $R(x)$, and moreover a common zero of $P(x, v)$, $Q(x, v)$, considered as polynomials of $x$ :

$$
P(\xi, v)=Q(\xi, v)=0 .
$$

Proof. Since $\eta$ has to be a root of $r(y)$, i.e. $r(\eta)=0$, we see that the system (17) admits a nontrivial solution. (Of course, in this system the point $(\xi, 1)$ is considered as an element of $\mathbb{C P}^{1}$, so that in the case $a(\eta)=c(\eta)=0$ it has to be taken equal to $(1,0)$.) First suppose that $(a(\eta), c(\eta)) \neq(0,0)$. Notice that there holds, identically in $u$,

$$
X(\eta, u)=\frac{d(\eta) u-b(\eta)}{-c(\eta) u+a(\eta)} \equiv \xi
$$

Substituting this into (14) we find:

$$
\begin{aligned}
& p(\eta, u)=(-c(\eta) u+a(\eta))^{2}(A(\xi) \eta+B(\xi)), \\
& q(\eta, u)=(-c(\eta) u+a(\eta))^{2}(C(\xi) \eta+D(\xi)),
\end{aligned}
$$

and these both polynomials in $u$ vanish identically, if and only if (18) holds. Now let $a(\eta)=c(\eta)=0$. This means that the corresponding $\xi=\infty$, so that the system (18) has to be read projectively, as:

$$
\tilde{A}(0) \eta+\tilde{B}(0)=0, \quad \tilde{C}(0) \eta+\tilde{D}(0)=0,
$$

where $\tilde{A}(x)=x^{2} A(1 / x)$, etc. At the same time, the formulas (14) have to be replaced by

$$
\begin{aligned}
& p(y, u)=(d(y) u-b(y))^{2}(\tilde{A}(1 / X) y+\tilde{B}(1 / X)), \\
& q(y, u)=(d(y) u-b(y))^{2}(\tilde{C}(1 / X) y+\tilde{D}(1 / X)),
\end{aligned}
$$


which yield

$$
\begin{aligned}
& p(\eta, u)=(d(\eta) u-b(\eta))^{2}(\tilde{A}(0) \eta+\tilde{B}(0)), \\
& q(\eta, u)=(d(\eta) u-b(\eta))^{2}(\tilde{C}(0) \eta+\tilde{D}(0)),
\end{aligned}
$$

and again these both polynomials in $u$ vanish identically, if and only if (20) holds. Finally, the statements about $\xi$ follow due to the symmetry of systems (17), (18) with respect to changing the roles of $x$ and $y$.

Notice that the system (17) characterizes the points $(\xi, \eta)$ where the first fraction in (3) is not defined. We see that for quadri-rational maps these points satisfy with necessity also the system (18) which characterizes the points where the second fraction in (3) is not defined. Due to Proposition 10 , we see that for quadri-rational maps singularities of both fractions in (3) coincide. Clearly, there are no more than four such singularities.

In what follows, we shall take the freedom of performing Möbius transformations in the variables $x, y, u, v$ in order not to consider various particular cases as in the previous proof. Indeed, performing a Möbius transformation in $x$, we can achieve that neither root $\xi$ of $R(x)$ lies at $\infty$, that is, $(a(\eta), c(\eta)) \neq(0,0)$ for all zeros $\eta$ of $r(y)$. Next, by doing a Möbius transformation in $u$, we can even assure that both $a(\eta) \neq 0, c(\eta) \neq 0$. Similarly, we shall assume that both $A(\xi) \neq 0, C(\xi) \neq 0$. Note also that under these conditions multiple zeros of the functions $p(y, u), q(y, u)$, considered as polynomials in $y$, can be alternatively characterized as zeros of the same multiplicity of the functions

$$
\tilde{p}(y, u)=A(X) y+B(X), \quad \tilde{q}(y, u)=C(X) y+D(X) .
$$

This is seen immediately from (14).

Proposition 11 (cancellation of double zero). In order for $\eta$ to be a common double zero of $p(y, u)$ and $q(y, u)$ (considered as polynomials in $y$ ), it is necessary and sufficient that the point $(\xi, \eta)$ satisfies, in addition to (17), (18), the following equations:

$$
\begin{aligned}
& \left\{\begin{array}{l}
a^{\prime}(\eta) \xi+b^{\prime}(\eta)+\lambda a(\eta)=0, \\
c^{\prime}(\eta) \xi+d^{\prime}(\eta)+\lambda c(\eta)=0,
\end{array}\right. \\
& \left\{\begin{array}{l}
A^{\prime}(\xi) \eta+B^{\prime}(\xi)+\lambda^{-1} A(\xi)=0, \\
C^{\prime}(\xi) \eta+D^{\prime}(\xi)+\lambda^{-1} C(\xi)=0,
\end{array}\right.
\end{aligned}
$$

with some $\lambda \in \mathbb{C P}^{1}$. Such $\eta$ is with necessity a double zero of $r(y)$, and the corresponding $\xi$ is a double zero of $R(x)$. Moreover, $\xi$ is a common double zero of $P(x, v)$ and $Q(x, v)$ (considered as polynomials in $x$ ). 
Proof. Differentiate the definitions (21) with respect to $y$, then set $y=\eta$, taking into account Eq. (19). The result reads:

$$
\begin{aligned}
& \left(A^{\prime}(\xi) \eta+B^{\prime}(\xi)\right) X_{y}(\eta, u)+A(\xi)=0, \\
& \left(C^{\prime}(\xi) \eta+D^{\prime}(\xi)\right) X_{y}(\eta, u)+C(\xi)=0 .
\end{aligned}
$$

Eqs. (24) can be fulfilled identically in $u$ if and only if $X_{y}(\eta, u)$ is constant, i.e. does not depend on $u$. Denote this constant by

$$
X_{y}(\eta, u) \equiv \lambda,
$$

then Eqs. (24) coincide with (23). Differentiate

$$
X(y, u)(c(y) u-a(y))+d(y) u-b(y)=0
$$

with respect to $y$ and set $y=\eta$. Taking into account that $X(\eta, u) \equiv \xi$ and $X_{y}(\eta, u) \equiv \lambda$, one comes to the formula

$$
\lambda(c u-a)+\xi\left(c^{\prime} u-a^{\prime}\right)+\left(d^{\prime} u-b^{\prime}\right)=0,
$$

where we write for shortness $a, a^{\prime}$ etc. for $a(\eta), a^{\prime}(\eta)$ etc. This is equivalent to (22). To prove that $\eta$ is a double root of $r(y)$, calculate:

$$
r^{\prime}(\eta)=a^{\prime} d+a d^{\prime}-b^{\prime} c-b c^{\prime}=-\left(a^{\prime} \xi+b^{\prime}\right) c+a\left(c^{\prime} \xi+d^{\prime}\right)=0 ;
$$

on the last step we used Eq. (22). The proof that $\xi$ is a double root of $R(x)$ is analogous, since the systems $(22),(23)$ are symmetric with respect to the interchange $\xi \leftrightarrow \eta$.

It will be convenient to represent the parameter $\lambda$ as a quotient:

$$
\lambda=\dot{\xi} / \dot{\eta}
$$

Clearly, the quantities $\dot{\xi}, \dot{\eta}$ are defined only up to a common factor. They can be considered as representing the velocity vector at $(\xi, \eta)$ of a parametrized curve on the plane $\mathbb{C P}^{1} \times \mathbb{C P}^{1}$ passing through this point. The freedom of a simultaneous multiplying $\dot{\xi}$ and $\dot{\eta}$ by a common factor corresponds to a possibility to re-parametrize such a curve. This supports the intuitive understanding of the present case as a degeneration of the previous one, when two "simple singularities" glue together moving along some curve with 
a certain limiting velocity. This understanding is further supported by the form of the system $(22),(23)$ in this new notation:

$$
\begin{aligned}
& \left\{\begin{array}{l}
\left(a^{\prime}(\eta) \xi+b^{\prime}(\eta)\right) \dot{\eta}+a(\eta) \dot{\xi}=0, \\
\left(c^{\prime}(\eta) \xi+d^{\prime}(\eta)\right) \dot{\eta}+c(\eta) \dot{\xi}=0,
\end{array}\right. \\
& \left\{\begin{array}{l}
\left(A^{\prime}(\xi) \eta+B^{\prime}(\xi)\right) \dot{\xi}+A(\xi) \dot{\eta}=0, \\
\left(C^{\prime}(\xi) \eta+D^{\prime}(\xi)\right) \dot{\xi}+C(\xi) \dot{\eta}=0 .
\end{array}\right.
\end{aligned}
$$

This can be clearly read as the result of differentiation of $(17),(18)$ at $(\xi, \eta)$ along the parametrized curve through $(\xi, \eta)$ with the velocity vector $(\dot{\xi}, \dot{\eta})$.

Proposition 12 (cancellation of triple zero). In order for $\eta$ to be a common triple zero of $p(y, u)$ and $q(y, u)$ (considered as polynomials in $y$ ), it is necessary and sufficient that the point $(\xi, \eta)$ satisfies, in addition to (17), (18), (22), (23), the following equations:

$$
\begin{aligned}
& \left\{\begin{array}{l}
a^{\prime \prime}(\eta) \xi+b^{\prime \prime}(\eta)+2 \lambda a^{\prime}(\eta)+\lambda^{3 / 2} \theta a(\eta)=0, \\
c^{\prime \prime}(\eta) \xi+d^{\prime \prime}(\eta)+2 \lambda c^{\prime}(\eta)+\lambda^{3 / 2} \theta c(\eta)=0,
\end{array}\right. \\
& \left\{\begin{array}{l}
A^{\prime \prime}(\xi) \eta+B^{\prime \prime}(\xi)+2 \lambda^{-1} A^{\prime}(\xi)-\lambda^{-3 / 2} \theta A(\xi)=0, \\
C^{\prime \prime}(\xi) \eta+D^{\prime \prime}(\xi)+2 \lambda^{-1} C^{\prime}(\xi)-\lambda^{-3 / 2} \theta C(\xi)=0,
\end{array}\right.
\end{aligned}
$$

with some $\theta \in \mathbb{C P}^{1}$. Such $\eta$ is with necessity a triple zero of $r(y)$, and the corresponding $\xi$ is a triple zero of $R(x)$. Moreover, $\xi$ is a common triple zero of $P(x, v)$ and $Q(x, v)$ (considered as polynomials in $x$ ).

Proof. Differentiate (21) twice with respect to $y$, then set $y=\eta$, taking into account Eq. (19). The result reads:

$$
\begin{array}{r}
\left(A^{\prime \prime}(\xi) \eta+B^{\prime \prime}(\xi)\right) X_{y}^{2}(\eta, u)+2 A^{\prime}(\xi) X_{y}(\eta, u) \\
+\left(A^{\prime}(\xi) \eta+B^{\prime}(\xi)\right) X_{y y}(\eta, u)=0 \\
\left(C^{\prime \prime}(\xi) \eta+D^{\prime \prime}(\xi)\right) X_{y}^{2}(\eta, u)+2 C^{\prime}(\xi) X_{y}(\eta, u) \\
+\left(C^{\prime}(\xi) \eta+D^{\prime}(\xi)\right) X_{y y}(\eta, u)=0 .
\end{array}
$$

Using (23) and the value $X_{y}(\eta, u)=\lambda$ from (25), we bring this into the form

$$
\begin{aligned}
& A^{\prime \prime}(\xi) \eta+B^{\prime \prime}(\xi)+2 \lambda^{-1} A^{\prime}(\xi)-\lambda^{-3} A(\xi) X_{y y}(\eta, u)=0, \\
& C^{\prime \prime}(\xi) \eta+D^{\prime \prime}(\xi)+2 \lambda^{-1} C^{\prime}(\xi)-\lambda^{-3} C(\xi) X_{y y}(\eta, u)=0 .
\end{aligned}
$$

Eqs. (32) can be fulfilled identically in $u$ if and only if $X_{y y}(\eta, u)$ does not depend on $u$; we denote this constant by

$$
X_{y y}(\eta, u) \equiv \lambda^{3 / 2} \theta,
$$


and then (32) coincide with (31). Next, differentiate (26) twice with respect to $y$ and set $y=\eta$. Taking into account Eqs. (19), (25) and (33), one finds:

$$
\lambda^{3 / 2} \theta(c u-a)+2 \lambda\left(c^{\prime} u-a^{\prime}\right)+\xi\left(c^{\prime \prime} u-a^{\prime \prime}\right)+\left(d^{\prime \prime} u-b^{\prime \prime}\right)=0 .
$$

This is equivalent to (30). It remains to prove that $\eta$ is indeed a triple root of $r(y)$. For this, note that under the conditions (17), (22), we have:

$$
\begin{aligned}
r^{\prime \prime}(\eta) & =a^{\prime \prime} d+2 a^{\prime} d^{\prime}+a d^{\prime \prime}-b^{\prime \prime} c-2 b^{\prime} c^{\prime}-b c^{\prime \prime} \\
& =-\left(a^{\prime \prime} \xi+b^{\prime \prime}\right) c+\left(c^{\prime \prime} \xi+d^{\prime \prime}\right) a+2 a^{\prime}\left(-c^{\prime} \xi-\lambda c\right)-2 c^{\prime}\left(-a^{\prime} \xi-\lambda a\right) \\
& =-\left(a^{\prime \prime} \xi+b^{\prime \prime}+2 \lambda a^{\prime}\right) c+\left(c^{\prime \prime} \xi+d^{\prime \prime}+2 \lambda c^{\prime}\right) a,
\end{aligned}
$$

and this vanishes due to (30).

It will be convenient to parametrize $\theta$ by the $2-$ germ of a curve through $(\xi, \eta)$, similar to the parametrization $(27)$ of $\lambda$ by the 1 -germ. To find such a parametrization, differentiate the relations (17), (18) twice along a (parametrized) curve through $(\xi, \eta)$ with the 2 -germ at this point given by $(\dot{\xi}, \ddot{\xi}, \dot{\eta}, \ddot{\eta})$ :

$$
\begin{aligned}
& \left\{\begin{array}{l}
\left(a^{\prime \prime}(\eta) \xi+b^{\prime \prime}(\eta)\right) \dot{\eta}^{2}+2 a^{\prime}(\eta) \dot{\xi} \dot{\eta}+\left(a^{\prime}(\eta) \xi+b^{\prime}(\eta)\right) \ddot{\eta}+a(\eta) \ddot{\xi}=0, \\
\left(c^{\prime \prime}(\eta) \xi+d^{\prime \prime}(\eta)\right) \dot{\eta}^{2}+2 c^{\prime}(\eta) \dot{\xi} \dot{\eta}+\left(c^{\prime}(\eta) \xi+d^{\prime}(\eta)\right) \ddot{\eta}+c(\eta) \ddot{\xi}=0,
\end{array}\right. \\
& \left\{\begin{array}{l}
\left(A^{\prime \prime}(\xi) \eta+B^{\prime \prime}(\xi)\right) \dot{\xi}^{2}+2 A^{\prime}(\xi) \dot{\xi} \dot{\eta}+\left(A^{\prime}(\xi) \eta+B^{\prime}(\xi)\right) \ddot{\xi}+A(\xi) \ddot{\eta}=0, \\
\left(C^{\prime \prime}(\xi) \eta+D^{\prime \prime}(\xi)\right) \dot{\xi}^{2}+2 C^{\prime}(\xi) \dot{\xi} \dot{\eta}+\left(C^{\prime}(\xi) \eta+D^{\prime}(\xi)\right) \ddot{\xi}+C(\xi) \ddot{\eta}=0 .
\end{array}\right.
\end{aligned}
$$

Taking into account Eqs. (28) and (27), one sees that the above relations can be written as $(30),(31)$, respectively, provided

$$
\theta=\frac{\ddot{\xi} \dot{\eta}-\dot{\xi} \ddot{\eta}}{(\dot{\xi} \dot{\eta})^{3 / 2}} .
$$

This representation is actually independent of the parametrization of the curve, and respects the symmetry $\theta \rightarrow-\theta$ as $\xi \leftrightarrow \eta$, as it should.

Proposition 13 (cancellation of quadruple zero). In order for $\eta$ to be a common quadruple zero of $p(y, u)$ and $q(y, u)$ (considered as polynomials in $y)$, it is necessary and sufficient that the point $(\xi, \eta)$ satisfies, in addition to (17), (18), (22), (23), (30), (31), the following equations:

$$
\begin{aligned}
& \left\{\begin{array}{l}
a^{\prime \prime}(\eta)+\lambda^{1 / 2} \theta a^{\prime}(\eta)+\frac{1}{2} \lambda\left(\theta^{2}+\varkappa\right) a(\eta)=0, \\
c^{\prime \prime}(\eta)+\lambda^{1 / 2} \theta c^{\prime}(\eta)+\frac{1}{2} \lambda\left(\theta^{2}+\varkappa\right) c(\eta)=0,
\end{array}\right. \\
& \left\{\begin{array}{l}
A^{\prime \prime}(\xi)-\lambda^{-1 / 2} \theta A^{\prime}(\xi)+\frac{1}{2} \lambda^{-1}\left(\theta^{2}-\varkappa\right) A(\xi)=0, \\
C^{\prime \prime}(\xi)-\lambda^{-1 / 2} \theta C^{\prime}(\xi)+\frac{1}{2} \lambda^{-1}\left(\theta^{2}-\varkappa\right) C(\xi)=0,
\end{array}\right.
\end{aligned}
$$


with some $\varkappa \in \mathbb{C P}^{1}$. Such $\eta$ is with necessity a quadruple zero of $r(y)$, and the corresponding $\xi$ is a quadruple zero of $R(x)$. Moreover, $\xi$ is a common quadruple zero of $P(x, v)$ and $Q(x, v)$ (considered as polynomials in $x$ ).

Proof. Differentiate (21) thrice with respect to $y$, then set $y=\eta$, taking into account Eq. (19). The result reads:

$$
\begin{aligned}
\left(A^{\prime \prime}(\xi) \eta+B^{\prime \prime}(\xi)\right) X_{y}(\eta, u) X_{y y}(\eta, u)+A^{\prime}(\xi) X_{y y}(\eta, u)+A^{\prime \prime}(\xi) X_{y}^{2}(\eta, u) & \\
+\frac{1}{3}\left(A^{\prime}(\xi) \eta+B^{\prime}(\xi)\right) X_{y y y}(\eta, u) & =0, \\
\left(C^{\prime \prime}(\xi) \eta+D^{\prime \prime}(\xi)\right) X_{y}(\eta, u) X_{y y}(\eta, u)+C^{\prime}(\xi) X_{y y}(\eta, u)+C^{\prime \prime}(\xi) X_{y}^{2}(\eta, u) & \\
+\frac{1}{3}\left(C^{\prime}(\xi) \eta+D^{\prime}(\xi)\right) X_{y y y}(\eta, u) & =0 .
\end{aligned}
$$

Using (23), (31), and the values $X_{y}(\eta, u)=\lambda, X_{y y}(\eta, u)=\lambda^{3 / 2} \theta$ from (25), (33), we bring this into the form

$$
\begin{aligned}
& A^{\prime \prime}(\xi)-\lambda^{-1 / 2} \theta A^{\prime}(\xi)+\lambda^{-1} \theta^{2} A(\xi)-\frac{1}{3} \lambda^{-3} A(\xi) X_{y y y}(\eta, u)=0, \\
& C^{\prime \prime}(\xi)-\lambda^{-1 / 2} \theta C^{\prime}(\xi)+\lambda^{-1} \theta^{2} C(\xi)-\frac{1}{3} \lambda^{-3} C(\xi) X_{y y y}(\eta, u)=0 .
\end{aligned}
$$

Eqs. (39) are fulfilled identically in $u$ if and only if $X_{y y y}(\eta, u)$ does not depend on $u$. Denote this constant by

$$
X_{y y y}(\eta, u) \equiv \frac{3 \lambda^{2}}{2}\left(\theta^{2}+\varkappa\right),
$$

Then (39) are equivalent to (38). Next, differentiate (26) thrice with respect to $y$ and set $y=\eta$. Taking into account (19), (25), (33) and (40), we find:

$$
\frac{1}{2} \lambda^{2}\left(\theta^{2}+\varkappa\right)(c u-a)+\lambda^{3 / 2} \theta\left(c^{\prime} u-a^{\prime}\right)+\lambda\left(c^{\prime \prime} u-a^{\prime \prime}\right)=0,
$$

which is equivalent to (37). It remains to prove that $\eta$ is indeed a quadruple root of $r(y)$. For this, note:

$$
\begin{aligned}
\frac{1}{3} r^{\prime \prime \prime}(\eta) & =a^{\prime \prime} d^{\prime}+a^{\prime} d^{\prime \prime}-b^{\prime \prime} c^{\prime}-b^{\prime} c^{\prime \prime} \\
& =a^{\prime \prime} d^{\prime}-a^{\prime}\left(c^{\prime \prime} \xi+2 \lambda c^{\prime}+\lambda^{3 / 2} \theta c\right)+\left(a^{\prime \prime} \xi+2 \lambda a^{\prime}+\lambda^{3 / 2} \theta a\right) c^{\prime}-b^{\prime} c^{\prime \prime} \\
& =a^{\prime \prime}\left(c^{\prime} \xi+d^{\prime}\right)-c^{\prime \prime}\left(a^{\prime} \xi+b^{\prime}\right)+\lambda^{3 / 2} \theta\left(a c^{\prime}-a^{\prime} c\right) \\
& =-\lambda c\left(a^{\prime \prime}+\lambda^{1 / 2} \theta a^{\prime}\right)+\lambda a\left(c^{\prime \prime}+\lambda^{1 / 2} \theta c^{\prime}\right)=0 .
\end{aligned}
$$

In this computation we used subsequently (30), (22), (37).

Like in the cases of double and triple zeros (cf. (27), (36)), it will be convenient to parametrize $\varkappa$ by the 3 -germ of a curve through $(\xi, \eta)$. To 
find the corresponding formula, differentiate the relations (17), (18) thrice along a (parametrized) curve through $(\xi, \eta)$ with the 3 -germ at this point given by $(\dot{\xi}, \ddot{\xi}, \dddot{\xi}, \dot{\eta}, \ddot{\eta}, \dddot{\eta})$ :

$$
\begin{gathered}
\left\{\begin{array}{r}
\left(a^{\prime \prime}(\eta) \xi+b^{\prime \prime}(\eta)\right) \ddot{\eta} \dot{\eta}+a^{\prime \prime}(\eta) \dot{\xi} \dot{\eta}^{2}+a^{\prime}(\eta)(\ddot{\xi} \dot{\eta}+\dot{\xi} \ddot{\eta}) \\
+\frac{1}{3}\left(\left(a^{\prime}(\eta) \xi+b^{\prime}(\eta)\right) \dddot{\eta}+a(\eta) \dddot{\xi}\right)=0, \\
\left(c^{\prime \prime}(\eta) \xi+d^{\prime \prime}(\eta)\right) \ddot{\eta} \dot{\eta}+c^{\prime \prime}(\eta) \dot{\xi} \dot{\eta}^{2}+c^{\prime}(\eta)(\ddot{\xi} \dot{\eta}+\dot{\xi} \ddot{\eta}) \\
+\frac{1}{3}\left(\left(c^{\prime}(\eta) \xi+d^{\prime}(\eta)\right) \dddot{\eta}+c(\eta) \ddot{\xi}\right)=0
\end{array}\right. \\
\left\{\begin{array}{r}
\left(A^{\prime \prime}(\xi) \eta+B^{\prime \prime}(\xi)\right) \ddot{\xi} \dot{\xi}+A^{\prime \prime}(\xi) \dot{\xi}^{2} \dot{\eta}+A^{\prime}(\xi)(\ddot{\xi} \dot{\eta}+\dot{\xi} \ddot{\eta}) \\
+\frac{1}{3}\left(\left(A^{\prime}(\xi) \eta+B^{\prime}(\xi)\right) \ddot{\xi}+A(\xi) \dddot{\eta}\right)=0, \\
\left(C^{\prime \prime}(\xi) \eta+D^{\prime \prime}(\xi)\right) \ddot{\xi} \dot{\xi}+C^{\prime \prime}(\xi) \dot{\xi}^{2} \dot{\eta}+C^{\prime}(\xi)(\ddot{\xi} \dot{\eta}+\dot{\xi} \ddot{\eta}) \\
+\frac{1}{3}\left(\left(C^{\prime}(\xi) \eta+D^{\prime}(\xi)\right) \ddot{\xi}+D(\xi) \dddot{\eta}\right)=0 .
\end{array}\right.
\end{gathered}
$$

Taking into account Eqs. (28), (34) and (27), (36), one sees that the above relations can be written as $(37),(38)$, respectively, provided

$$
\varkappa=\frac{2(\dddot{\xi} \dot{\eta}-\dot{\xi} \dddot{\eta})}{3(\dot{\xi} \dot{\eta})^{2}}-\frac{\ddot{\xi}^{2} \dot{\eta}^{2}-\dot{\xi}^{2} \ddot{\eta}^{2}}{(\dot{\xi} \dot{\eta})^{3}}=\frac{2}{3 \dot{\xi} \dot{\eta}}(S(\xi)-S(\eta)),
$$

where $S(\xi)$ stands for the Schwarzian derivative, $S(\xi)=\dddot{\xi} / \dot{\xi}-3 \ddot{\xi}^{2} / 2 \dot{\xi}^{2}$. Like (36), representation (41) is actually independent of the parametrization of the curve, and respects the symmetry $\varkappa \rightarrow-\varkappa$ as $\xi \leftrightarrow \eta$, as it should.

\section{Five types of quadrirational maps.}

A polynomial $R(x)$ of the 4 th degree belongs to one of the five types, $\mathcal{T}=$ $\mathrm{I}, \ldots, \mathrm{V}$, according to the multiplicity of zeros, as shown in the second column of the table 1 . We will denote by $m$ the number of the distinct roots, given in the third column. The ordering of the roots with respect to their multiplicity will be firmly associated to the type of the polynomial. Note that we consider the polynomials projectively, with the action of the Möbius transformations given by $R(x) \mapsto(\gamma x+\delta)^{4} R((\alpha x+\beta) /(\gamma x+\delta))$. Hence, for instance, the polynomial $x(x-1)$ considered as the polynomial of the 4 th degree is of the type II, having two simple roots $x_{1}=0, x_{2}=1$ and one double root $x_{3}=\infty$.

Theorem 14. Consider a bi-Möbius map $F$ defined by (6) with $\operatorname{deg}_{y} \phi=$ $\operatorname{deg}_{x} \Phi=2$. Necessary and sufficient conditions for $F$ to be quadrirational read: 


\begin{tabular}{|c|c|c|c|c|c|}
\hline $\mathcal{T}$ & zeroes & $m$ & $\mathcal{D}_{\mathcal{T}}(x)$ & $q_{\mathcal{T}}(x)$ & $\mathcal{C}_{\mathcal{T}}(\alpha)$ \\
\hline I & $\left(x_{1}, x_{2}, x_{3}, x_{4}\right)$ & 4 & $\left(x_{1}, x_{2}, x_{3}, x_{4}\right)$ & $\frac{\left(x_{1}-x_{2}\right)\left(x_{3}-x_{4}\right)}{\left(x_{2}-x_{3}\right)\left(x_{4}-x_{1}\right)}$ & $(\infty, 1,0, \alpha)$ \\
II & $\left(x_{1}, x_{2}, x_{3}, x_{3}\right)$ & 3 & $\left(x_{1}, x_{2}, x_{3}, \dot{x}_{3}\right)$ & $\frac{\dot{x}_{3}\left(x_{1}-x_{2}\right)}{\left(x_{2}-x_{3}\right)\left(x_{1}-x_{3}\right)}$ & $(\infty, 1,0, \alpha)$ \\
III & $\left(x_{1}, x_{1}, x_{2}, x_{2}\right)$ & 2 & $\left(x_{1}, \dot{x}_{1}, x_{2}, \dot{x}_{2}\right)$ & $\frac{\dot{x}_{1} \dot{x}_{2}}{\left(x_{1}-x_{2}\right)^{2}}$ & $(1,1,0, \alpha)$ \\
IV & $\left(x_{1}, x_{2}, x_{2}, x_{2}\right)$ & 2 & $\left(x_{1}, x_{2}, \dot{x}_{2}, \ddot{x}_{2}\right)$ & $\frac{\ddot{x}_{2}}{2 \dot{x}_{2}}+\frac{\dot{x}_{2}}{x_{1}-x_{2}}$ & $(\infty, 0,1,2 \alpha)$ \\
V & $\left(x_{1}, x_{1}, x_{1}, x_{1}\right)$ & 1 & $\left(x_{1}, \dot{x}_{1}, \ddot{x}_{1}, \dddot{x}_{1}\right)$ & $\frac{1}{6}\left(\frac{\dddot{x}_{1}}{\dot{x}_{1}}-\frac{3 \ddot{x}_{1}^{2}}{2 \dot{x}_{1}^{2}}\right)$ & $(0,1,0,6 \alpha)$ \\
\hline
\end{tabular}

Table 1: Möbius invariants and canonical data

- $R(x)$ and $r(y)$ belong simultaneously to the same type $\mathcal{T}$;

- The roots $x_{i}, y_{i}(i=1, \ldots, m)$ of $R(x), r(y)$ can be ordered so that:

1. there hold (17), (18) with $(\xi, \eta)=\left(x_{i}, y_{i}\right)$ for all $i=1, \ldots, m$;

2. for roots $x_{i}, y_{i}$ of multiplicity $\geq 2$ there hold additionally (22), (23) with $(\xi, \eta)=\left(x_{i}, y_{i}\right)$ and with some $\lambda=\lambda_{i} \in \mathbb{C P}^{1}$;

3. for roots $x_{i}, y_{i}$ of multiplicity $\geq 3$ there hold additionally (30), (31) with $(\xi, \eta)=\left(x_{i}, y_{i}\right), \lambda=\lambda_{i}$, and with some $\theta=\theta_{i} \in \mathbb{C P}^{1}$;

4. for quadruple roots $x_{i}, y_{i}$ there hold additionally (37), (38) with $(\xi, \eta)=\left(x_{i}, y_{i}\right), \lambda=\lambda_{i}, \theta=\theta_{i}$ and with some $\varkappa=\varkappa_{i} \in \mathbb{C P}^{1}$.

Proof. This follows directly from Propositions 10, 11, 12, 13: we see that the polynomial $\rho(y)$ in (15) has to coincide with $r(y)$, while the polynomial $\mathrm{P}(x)$ in (16) has to coincide with $R(x)$, and, moreover, both polynomials have to belong simultaneously to one of the types I-V.

Definition 15. We say that a quadrirational map $F$ defined by (6) with $\operatorname{deg}_{y} \phi=\operatorname{deg}_{x} \Phi=2$ is of type $\mathcal{T}$ if the polynomials $R(x)$ and $r(y)$ are of type $\mathcal{T}$. 
The formulas

$$
\lambda_{i}=\frac{\dot{x}_{i}}{\dot{y}_{i}}, \quad \theta_{i}=\frac{\ddot{x}_{i} \dot{y}_{i}-\dot{x}_{i} \ddot{y}_{i}}{\left(\ddot{x}_{i} \dot{y}_{i}\right)^{3 / 2}}, \quad \varkappa_{i}=\frac{2}{3 \dot{x}_{i} \dot{y}_{i}}\left(S\left(x_{i}\right)-S\left(y_{i}\right)\right)
$$

allow us to describe the singularities of a quadrirational map by associating the data $\mathcal{D}_{\mathcal{T}}(x), \mathcal{D}_{\mathcal{T}}(y)$ to the edges $x, y$ of the elementery quadrilateral. These data are shown in the fourth column of the Table 1,

In all cases except for $\mathcal{T}=$ I these data are defined not uniquely, but up to transformations

$$
x_{i}^{\prime}=\dot{x}_{i} \gamma_{1}, \quad x_{i}^{\prime \prime}=\ddot{x}_{i} \gamma_{1}^{2}+\dot{x}_{i} \gamma_{2}, \quad x_{i}^{\prime \prime \prime}=\dddot{x}_{i} \gamma_{1}^{3}+3 \ddot{x}_{i} \gamma_{2} \gamma_{1}+\dot{x}_{i} \gamma_{3}
$$

and similarly for the edge $y$, with the same $\gamma_{1}, \gamma_{2}, \gamma_{3}$. In the only case $\mathcal{T}=$ III when such transformations are applicable with two different values $i=1,2$, the quantities $\gamma_{1}$ can be chosen for these $i$ independently (but for each $i=1,2$ they still have to be the same for the edges $x$ and $y$ ). These quantities $\gamma_{1}, \gamma_{2}, \gamma_{3}$ have the meaning of

$$
\gamma_{1}=t^{\prime}, \quad \gamma_{2}=t^{\prime \prime}, \quad \gamma_{3}=t^{\prime \prime \prime}
$$

for the change of "time" $t=t(s)$ in the parametrized curves $(x(t), y(t))$ through $\left(x_{i}, y_{i}\right)$ etc. Clearly, the values of the parameters (42) do not change under transformations (43).

The action of Möbius transformations $x \mapsto(\alpha x+\beta) /(\gamma x+\delta)$ on the data $\mathcal{D}_{\mathcal{T}}$ is defined by the natural formulas, obtained simply by differentiation:

$$
x_{i} \mapsto \frac{\alpha x_{i}+\beta}{\gamma x_{i}+\delta}, \quad \dot{x}_{i} \mapsto \frac{(\alpha \delta-\beta \gamma) \dot{x}_{i}}{\left(\gamma x_{i}+\delta\right)^{2}}, \quad \text { etc. }
$$

Proposition 16. The data $\mathcal{D}_{\mathcal{T}}(x)$ admit the Möbius invariants $q_{\mathcal{T}}(x)$, as shown in the fifth column of the Table 1.

Here we take the freedom to use the abbreviation $q_{\mathcal{T}}(x)$ instead of the more correct notation $q_{\mathcal{T}}\left(\mathcal{D}_{\mathcal{T}}(x)\right)$. The statement is proved by straightforward computation. This is, of course, well known for $\mathcal{T}=\mathrm{I}$ and $\mathrm{V}$, where $q_{\mathrm{I}}$ is the classical cross-ratio, and $q_{\mathrm{V}}$ coincides, up to $1 / 6$, with the Schwarzian derivative.

The presented invariants are complete in the following sense: two data of the same type $\mathcal{T}$ are Möbius equivalent if and only if the values of the corresponding invariants coincide. In particular, for any data $\mathcal{D}_{\mathcal{T}}(x)$ with the value of the invariant $q_{\mathcal{T}}(x)=\alpha$ there exists a unique Möbius transformation 
bringing it into the canonical representative $\mathcal{D}_{\mathcal{T}}=\mathcal{C}_{\mathcal{T}}(\alpha)$, shown in the last column of the Table 1.

Finally, we notice that the above hierarchy of Möbius invariants may be obtained by the following series of natural degeneration processes:

$\mathrm{I} \rightarrow \mathrm{II}:$ let $x_{4}=x_{3}+\epsilon \dot{x}_{3}+O\left(\epsilon^{2}\right)$, then

$$
q_{\mathrm{I}}\left(x_{1}, x_{2}, x_{3}, x_{4}\right)=\epsilon q_{\mathrm{II}}\left(x_{1}, x_{2}, x_{3}, \dot{x}_{3}\right) ;
$$

II $\rightarrow$ III: let $x_{2}=x_{1}+\epsilon \dot{x}_{1}+O\left(\epsilon^{2}\right)$, then

$$
q_{\mathrm{II}}\left(x_{1}, x_{2}, x_{3}, \dot{x}_{3}\right)=\epsilon q_{\mathrm{III}}\left(x_{1}, \dot{x}_{1}, x_{3}, \dot{x}_{3}\right) \text {; }
$$

$\mathrm{II} \rightarrow \mathrm{IV}:$ let $x_{3}=x_{2}+\epsilon \dot{x}_{2}+\epsilon^{2} \ddot{x}_{2} / 2+O\left(\epsilon^{3}\right)$, then

$$
q_{\mathrm{II}}\left(x_{1}, x_{2}, x_{3}, \dot{x}_{3}\right)=-\epsilon^{-1}\left(1+\epsilon q_{\mathrm{IV}}\left(x_{1}, x_{2}, \dot{x}_{2}, \ddot{x}_{2}\right)\right) ;
$$

$\mathrm{IV} \rightarrow \mathrm{V}:$ let $x_{2}=x_{1}+\epsilon \dot{x}_{1}+\epsilon^{2} \ddot{x}_{1} / 2+\epsilon^{3} \dddot{x}_{1} / 6+O\left(\epsilon^{4}\right)$, then

$$
q_{\mathrm{IV}}\left(x_{1}, x_{2}, \dot{x}_{2}, \ddot{x}_{2}\right)=-\epsilon^{-1}\left(1-\epsilon q_{\mathrm{V}}\left(x_{1}, \dot{x}_{1}, \ddot{x}_{1}, \dddot{x}_{1}\right)\right) .
$$

\section{Characterization of quadrirational maps by singularities patterns.}

The following statement is an easy corollary of Theorem 14 .

Proposition 17. For a quadrirational map $F$ of type $\mathcal{T}$ the polynomials $\widehat{R}(u), \widehat{r}(v)$ also belong to the type $\mathcal{T}$. Their roots $u_{i}, v_{i}(i=1, \ldots, m)$ can be ordered so that the companion map $\bar{F}:(u, y) \mapsto(x, v)$ has $m$ singularities $\left(u_{i}, y_{i}\right)$, the companion map $\bar{F}^{-1}:(x, v) \mapsto(u, y)$ has $m$ singularities $\left(x_{i}, v_{i}\right)$, and the inverse map $F^{-1}:(u, v) \mapsto(x, y)$ has $m$ singularities $\left(u_{i}, v_{i}\right)$, all three maps belonging to the type $\mathcal{T}$, as well.

Proof. The fact that the $y$-components of the singularities $\left(u_{i}, y_{i}\right)$ coincide with the $y$-components of the singularities $\left(x_{i}, y_{i}\right)$, follows from the fact that they also are the roots of the polynomial $r(y)$. Indeed, the formulas $(7)$ for the companion map yield:

$$
d\left(y_{i}\right) u_{i}-b\left(y_{i}\right)=0, \quad-c\left(y_{i}\right) u_{i}+a\left(y_{i}\right)=0, \quad i=1, \ldots, m .
$$

Similarly, the $x$-components of the singularities $\left(x_{i}, v_{i}\right)$ coincide with the $x$-components of the singularities $\left(x_{i}, y_{i}\right)$. For the proof of the claim about the singularities of $F^{-1}$ make four 90-degree rotations. 
Now we are in a position to formulate the main result which gives a complete classification of quadrirational maps of the subclass [2:2].

Theorem 18. Attach to each edge $x, y, u, v$ of the quadrilateral on Fig. 1 the data of one and the same type $\mathcal{T}$. The necessary and sufficient condition for the existence of a quadrirational map $F:(x, y) \mapsto(u, v)$ of type $\mathcal{T}$ with the singularities data $\left(\mathcal{D}_{\mathcal{T}}(x), \mathcal{D}_{\mathcal{T}}(y)\right)$ and such that the companion maps $\bar{F}:(u, y) \mapsto(x, v)$ and $\bar{F}^{-1}:(x, v) \mapsto(u, y)$ have the singularities data $\left(\mathcal{D}_{\mathcal{T}}(u), \mathcal{D}_{\mathcal{T}}(y)\right)$ and $\left(\mathcal{D}_{\mathcal{T}}(x), \mathcal{D}_{\mathcal{T}}(v)\right)$, respectively, reads:

$$
q_{\mathcal{T}}(x)=q_{\mathcal{T}}(u)=\alpha, \quad q_{\mathcal{T}}(y)=q_{\mathcal{T}}(v)=\beta, \quad \alpha \neq \beta .
$$

If this condition is satisfied, then the corresponding map is unique.

Proof. Necessity follows from Theorem 8. To prove the existence and uniqueness, one can compute the required map as follows. The coefficients of the polynomials $a(y), \ldots, d(y)$ have to satisfy the system of equations consisting of the applicable ones among (17), (22), (30) and (37), with $(\xi, \eta)=\left(x_{i}, y_{i}\right)$, $\lambda=\lambda_{i}, \theta=\theta_{i}$ and $\varkappa=\varkappa_{i}$ (cf. (42)), and also the analogous equations obtained by replacing $(a(y), b(y), c(y), d(y))$ by $(d(y),-b(y),-c(y), a(y))$, with $(\xi, \eta)=\left(u_{i}, y_{i}\right)$ and the corresponding parameters $\lambda=\mu_{i}, \theta=\vartheta_{i}$ and $\varkappa=\kappa_{i}$ of the companion map $\bar{F}$. This gives in all cases 16 homogeneous linear equations for 12 coefficients of the polynomials $a(y), \ldots, d(y)$. It is enough to study this system for the special case when canonical representatives of the singularities data $\mathcal{D}_{\mathcal{T}}$ are chosen on each edge $x, y, u, v$. The case by case study shows that this system always admits a unique (up to a common factor) solution; we give below more details for two extreme cases $\mathcal{T}=\mathrm{I}$ and $\mathcal{T}=\mathrm{V}$. Analogously, the coefficients of the polynomials $A(x), \ldots, D(x)$ are uniquely defined by the singularities data. The resulting canonical quadrirational maps of type $\mathcal{T}$ will be listed below.

Remarks. 1) It is important to notice that the parameters $\alpha, \beta$ attached to the edges received in our construction an intrinsic interpretation in terms of singularities of the map.

2) In case $\alpha=\beta$ the above mentioned systems also admit a unique solution, but the resulting map degenerates: $u=y, v=x$, thus having no singularities at all.

The canonical map of type $\mathcal{T}$ is characterized by the singularities data

$$
\mathcal{D}_{\mathcal{T}}(x)=\mathcal{D}_{\mathcal{T}}(u)=\mathcal{C}_{\mathcal{T}}(\alpha), \quad \mathcal{D}_{\mathcal{T}}(y)=\mathcal{D}_{\mathcal{T}}(v)=\mathcal{C}_{\mathcal{T}}(\beta)
$$

accordingly to the Table 1 . 
Type I. The system for the coefficients of the polynomials $a(y), \ldots, d(y)$ consists of 8 equations (17) with $(\xi, \eta)=\left(x_{i}, y_{i}\right), i=1, \ldots, 4$, and of 8 equations (44). For the canonical choice (46) of the singularities data it reads:

$$
\begin{aligned}
a_{2}=c_{2}=0, \quad b_{0}=d_{0} & =0, & a_{0}=b_{0}=0, & c_{2}=d_{2}=0 \\
a_{0}+a_{1}+b_{1}+b_{2} & =0, & d_{0}+d_{1}-b_{1}-b_{2} & =0 \\
c_{0}+c_{1}+d_{1}+d_{2} & =0, & -c_{0}-c_{1}+a_{1}+a_{2} & =0 \\
\left(a_{0}+a_{1} \beta\right) \alpha+\left(b_{1} \beta+b_{2} \beta^{2}\right) & =0, & \left(d_{0}+d_{1} \beta\right) \alpha-\left(b_{1} \beta+b_{2} \beta^{2}\right) & =0 \\
\left(c_{0}+c_{1} \beta\right) \alpha+\left(d_{1} \beta+d_{2} \beta^{2}\right) & =0, & -\left(c_{0}+c_{1} \beta\right) \alpha+\left(a_{1} \beta+a_{2} \beta^{2}\right) & =0
\end{aligned}
$$

This system admits a unique (up to a common factor) solution resulting exactly in the map $\left(F_{\mathrm{I}}\right)$.

Type II. The canonical map is

$$
u=\frac{\alpha y(x-y)}{\beta x(1-y)-\alpha y(1-x)}, \quad v=\frac{\beta x(x-y)}{\beta x(1-y)-\alpha y(1-x)} .
$$

It can be obtained from $\left(F_{\mathrm{I}}\right)$ by the rescaling $\alpha \mapsto \epsilon \alpha, \beta \mapsto \epsilon \beta$, and then sending $\epsilon \rightarrow 0$. Upon inverting all variables $x, y, u, v$, this map takes the form $\left(F_{\text {II }}\right)$ with two simple singularities at $(0,0),(1,1)$ and one double singularity at $(\infty, \infty)$.

Type III. The canonical map is

$$
u=\frac{\alpha y(x-y)}{\beta x-\alpha y+(\beta-\alpha)\left(y^{2}-2 y\right) x}, \quad v=\frac{\beta x(x-y)}{\beta x-\alpha y+(\beta-\alpha)\left(x^{2}-2 x\right) y} .
$$

Upon the Möbius transformation of variables $x=1 /\left(x^{\prime}-1\right)$, and similarly for $y, u, v$, this map takes the form $\left(F_{\text {III }}\right)$ with two double singularities at $(0,0)$ and $(\infty, \infty)$. Obviously, this can be obtained from $\left(F_{\text {II }}\right)$ by multiplying all variables $x, y, u, v$ by $T$ and then sending $T \rightarrow \infty$.

Type IV. The canonical map is

$$
u=\frac{y(x-y)}{x-y+(\alpha-\beta) x y}, \quad v=\frac{x(x-y)}{x-y+(\alpha-\beta) x y} .
$$

Upon inverting all variables $x, y, u, v$ it takes the form $\left(F_{\mathrm{IV}}\right)$ with a simple singularity at $(0,0)$ and a triple singularity at $(\infty, \infty)$ : 
Type V. The system for the coefficients of the polynomials $a(y), \ldots, d(y)$ consists of (17), (22), (30) and (37), with $(\xi, \eta)=\left(x_{1}, y_{1}\right), \lambda=1, \theta=0$ and $\varkappa=4(\alpha-\beta)$, and also the analogous equations obtained by replacing $(a(y), b(y), c(y), d(y))$ by $(d(y),-b(y),-c(y), a(y))$, with $(\xi, \eta)=\left(u_{i}, y_{i}\right)$ (with the same values of parameters $\lambda, \theta$ and $\varkappa$ ). This system reads:

$$
\begin{array}{ll}
b_{0}=d_{0}=0, & a_{0}=b_{0}=0, \\
a_{0}+b_{1}=0, & d_{0}-b_{1}=0, \\
c_{0}+d_{1}=0, & a_{1}-c_{0}=0, \\
b_{2}+a_{1}=0, & d_{1}-b_{2}=0 \\
d_{2}+c_{1}=0, & a_{2}-c_{1}=0 \\
a_{2}+(\alpha-\beta) a_{0}=0, & d_{2}+(\alpha-\beta) d_{0}=0 \\
c_{2}+(\alpha-\beta) c_{0}=0, & c_{2}+(\alpha-\beta) c_{0}=0,
\end{array}
$$

and admits a unique (up to a common factor) solution resulting in the map

$$
u=\frac{y(x-y)}{x-y+(\beta-\alpha) x y^{2}}, \quad v=\frac{x(x-y)}{x-y+(\beta-\alpha) x^{2} y} .
$$

Upon inverting all variables $x, y, u, v$, it takes the form $\left(F_{\mathrm{V}}\right)$ with quadruple singularity at $(\infty, \infty)$.

\section{3D consistency and Lax representations.}

Theorem 19. Let each edge of the three-dimensional cube on Fig. 3 carry the data of the same type $\mathcal{T}$, such that the Möbius invariants $q_{\mathcal{T}}$ of the data on the opposite edges coincide. Attach quadrirational maps of type $\mathcal{T}$ to each face of the cube, according to Theorem 18. Then this system of maps is three-dimensionally consistent.

Proof. Performing a suitable Möbius transformation on each edge, we can assume that all edges carry the canonical representatives of their type. Then all maps on all faces are canonical. Consistency in this situation follows from Theorem 5 and the fact that all canonical maps $F_{\mathcal{T}}$ come from the geometric construction of Sect. 2. One might prefer a more direct proof. It can be (and has been) obtained by straightforward computations with the help of a symbolic computations system (we used Mathematica).

It is anticipated that the 3D consistency might be also proved with the help of a more deep study of the algebraic geometry of the maps $F_{\mathcal{T}}$. In this 
respect, an information on the blow-up and blow-down structure of these maps may be useful. Such information is provided in Appendices 1, 2.

As postulated in [2], the 3D consistency of discrete 2D systems can be taken as a definition of their integrability. In particular, one can derive from the $3 \mathrm{D}$ consistency such more traditional attributes of integrability as Lax representations. The correspondent procedure was elaborated in [2] for discrete models with fields on vertices, and in [14] for discrete models with fields on edges, i.e. for Yang-Baxter maps. The latter procedure looks as follows. Consider the parameter-dependent Yang-Baxter map $R(\alpha, \beta)$ : $(x, y) \mapsto(u, v)$. Suppose that on the set $\mathcal{X}$ there is an effective action of the linear group $G=G L_{N}$, and that the map $R(\alpha, \beta)$ has the following special form:

$$
u=M(y, \beta, \alpha)[x], \quad v=L(x, \alpha, \beta)[y],
$$

where $L, M: \mathcal{X} \times \mathbb{C} \times \mathbb{C} \mapsto G L_{N}$ are some matrix-valued functions on $\mathcal{X}$ depending on parameters $\alpha$ and $\beta$, and $M[x]$ denotes the action of $M \in G$ on $x \in \mathcal{X}$. Then the following relations hold:

$$
\begin{aligned}
L(x, \alpha, \lambda) L(y, \beta, \lambda) & =L(v, \beta, \lambda) L(u, \alpha, \lambda), \\
M(y, \beta, \lambda) M(x, \alpha, \lambda) & =M(u, \alpha, \lambda) M(v, \beta, \lambda) .
\end{aligned}
$$

Each one of these relations serves as a Lax representation for the map $R(\alpha, \beta)$.

In the present case of bi-Möbius maps (3) the above conditions are obviously satisfied with $G L_{2}$ matrices acting on $\mathbb{C P}^{1}$ by Möbius transformations,

$$
M(y)=\left(\begin{array}{ll}
a(y) & b(y) \\
c(y) & d(y)
\end{array}\right), \quad L(x)=\left(\begin{array}{ll}
A(x) & B(x) \\
C(x) & D(x)
\end{array}\right)
$$

The action is in this case projective, i.e. scalar matrices (and only they) are acting trivially, so that the resulting Lax representations are $\grave{a}$ priori only projective ones, i.e. (47) hold only up to a multiplication by scalars. But in each case it is not difficult to turn them into proper Lax representations by finding a suitable normalization.

Example. For the map $\left(F_{\mathrm{V}}\right)$ the Lax matrices obtained by the above procedure are:

$$
L(x, \alpha, \lambda)=\left(\begin{array}{cc}
x & \lambda-\alpha-x^{2} \\
1 & -x
\end{array}\right)
$$




\section{Concluding remarks.}

The notions and constructions of this paper can be generalized in various directions. One of them is the $(d+1)$-dimensional consistency of discrete $d$-dimensional systems, discussed in some detail in [2]. Another one deals with Yang-Baxter maps on more general varieties than $\mathbb{C P}^{1} \times \mathbb{C P}^{1}$, and we briefly discuss it now.

The construction in Sect. 2 leads to quadrirational maps on $\mathbb{C P}^{1} \times \mathbb{C P}^{1}$ after a rational parametrization of linear pencils of conics. It is not difficult to represent these maps also directly in terms of (non-homogeneous) coordinates on $\mathbb{C P}^{2}$. Consider a linear pencil of conics in $\mathbb{C}^{2}$ or $\mathbb{R}^{2}$ in the form

$$
Q_{\lambda}: \quad Q(X, \lambda)=\langle X,(\lambda S+T) X\rangle+\langle\lambda s+t, X\rangle+\lambda \sigma+\tau=0,
$$

where $S, T$ are symmetric matrices, $s, t$ are vectors and $\sigma, \tau$ scalars. Let $X \in Q_{\alpha}$ and $Y \in Q_{\beta}$. An easy computation shows that two other points of intersection of the line $\overline{X Y}$ with these conics are given by the formula

$$
\begin{aligned}
& X_{2}=Y+\frac{(\alpha-\beta)(\langle Y, S Y\rangle+\langle s, Y\rangle+\sigma)}{\langle X-Y,(\alpha S+T)(X-Y)\rangle}(X-Y), \\
& Y_{1}=X+\frac{(\alpha-\beta)(\langle X, S X\rangle+\langle s, X\rangle+\sigma)}{\langle X-Y,(\beta S+T)(X-Y)\rangle}(X-Y) .
\end{aligned}
$$

Of course, this formula can be rewritten in several ways using the relations $Q(X, \alpha)=Q(Y, \beta)=0$. However, the presented version has a remarkable property: it does not contain $t$ and $\tau$. This allows one to interpret the initial values $X, Y$ as free points of the plane, rather than points on the conics $Q_{\alpha}$ and $Q_{\beta}$, and the map $(X, Y) \rightarrow\left(X_{2}, Y_{1}\right)$ as a map $\mathbb{C}^{2} \times \mathbb{C}^{2} \rightarrow \mathbb{C}^{2} \times \mathbb{C}^{2}$ rather than a map $Q_{\alpha} \times Q_{\beta} \rightarrow Q_{\alpha} \times Q_{\beta}$.

Proposition 20. Formulas (49) define the $3 D$ consistent map $\mathbb{C}^{n} \times \mathbb{C}^{n} \rightarrow$ $\mathbb{C}^{n} \times \mathbb{C}^{n}$ for an arbitrary dimension $n$.

Proof. It is easy to see that all points $X_{2}, \ldots, Z_{12}$ taking part in the definition of $3 \mathrm{D}$ consistency will lie in the two-dimensional plane through the initial points $X, Y, Z$. Therefore, the proof can be effectively reduced to the case $n=2$. In this case, determine the vector $t$ and the scalar $\tau$ from the equations $Q(X, \alpha)=Q(Y, \beta)=Q(Z, \gamma)=0$. Obviously, these equations form a linear system for $t, \tau$, with the determinant equal to zero if and only 
if $X, Y$ and $Z$ lie on a straight line, which is a variety of codimension 1 . For $t, \tau$ thus found consider the linear pencil of conics (48) and apply Theorem 5 .

Note that the map (49) makes sense also in the scalar case $n=1$, where it coincides with one of the maps from the list $\left(F_{\mathrm{II}}\right)-\left(F_{\mathrm{V}}\right)$. For example, if $S \neq 0$ then we recover the map $\left(F_{\text {II }}\right)$, so that the formula $(49)$ can be considered as its multifield generalization.

Of course, there exist more 3D consistent maps in the multi-dimensional case. As another example we present the map

$X_{2}=-\frac{\beta}{\alpha} Y+\frac{\alpha-\beta}{\alpha}\left(X^{-1}-Y^{-1}\right)^{-1}, \quad Y_{1}=-\frac{\alpha}{\beta} X+\frac{\alpha-\beta}{\beta}\left(X^{-1}-Y^{-1}\right)^{-1}$,

which generalizes the scalar map $\left(F_{\text {III }}\right)$. Here $X, Y$ may be complex matrices or vectors, and $X^{-1}$ denotes the matrix inverse or the "vector inverse" $X^{-1}=$ $-X /|X|^{2}$, respectively.

There exist also maps without a direct scalar analog, for example those introduced in $[8,12]$. It should be stressed once more that in all known cases $3 \mathrm{D}$ consistent maps are quadrirational.

\section{Appendices.}

\section{Blow-down.}

As it happens with birational maps of two-dimensional varieties, our quadrirational maps blow up singular points to curves, and blow down exceptional curves to points.

Theorem 21. Consider a quadrirational map $F$ of type $\mathcal{T}$. Then there are exactly $m$ exceptional curves $\mathcal{C}_{i}, i=1, \ldots, m$, on $\mathbb{C P}^{1} \times \mathbb{C P}^{1}$, which are blown down by the map $F$ to the points $\left(u_{i}, v_{i}\right)$. The curves $\mathcal{C}_{i}=F^{-1}\left(u_{i}, v_{i}\right)$ have bidegree $(1,1)$ and are characterized by the following conditions.

I: In this case $m=4$. The curve $\mathcal{C}_{i}(1 \leq i \leq 4)$ passes through three points $\left(x_{j}, y_{j}\right)$ with $j \neq i$.

II: In this case $m=3$. For $i=1,2$, the curve $\mathcal{C}_{i}$ passes through two points $\left(x_{j}, y_{j}\right), j \neq i$, with the tangent at $\left(x_{3}, y_{3}\right)$ parallel to $\left(\dot{x}_{3}, \dot{y}_{3}\right)$. The curve $\mathcal{C}_{3}$ passes through all three points $\left(x_{j}, y_{j}\right)$.

III: In this case $m=2$. The curve $\mathcal{C}_{i}(i=1,2)$ passes through the both points $\left(x_{1}, y_{1}\right)$ and $\left(x_{2}, y_{2}\right)$, with the tangent at $\left(x_{j}, y_{j}\right), j \neq i$, parallel to $\left(\dot{x}_{j}, \dot{y}_{j}\right)$. 
$I V$ : In this case $m=2$. The curve $\mathcal{C}_{1}$ passes through the point $\left(x_{2}, y_{2}\right)$, and has there the 2-germ defined by $\left(\dot{x}_{2}, \dot{y}_{2}, \ddot{x}_{2}, \ddot{y}_{2}\right)$. The curve $\mathcal{C}_{2}$ passes through the both points $\left(x_{j}, y_{j}\right)$ with the tangent at $\left(x_{2}, y_{2}\right)$ parallel to $\left(\dot{x}_{2}, \dot{y}_{2}\right)$.

$V$ : In this case $m=1$. The curve $\mathcal{C}_{1}$ passes through the point $\left(x_{1}, y_{1}\right)$ and has there the 2-germ defined by $\left(\dot{x}_{1}, \dot{y}_{1}, \ddot{x}_{1}, \ddot{y}_{1}\right)$.

Proof. It is clear (and well known) that only singular points $\left(u_{i}, v_{i}\right)$ can be images of exceptional curves. Fix $1 \leq i \leq m$; the pre-image $\mathcal{C}_{i}=F^{-1}\left(u_{i}, v_{i}\right)$ is described by the equations

$$
\varphi\left(x, y, u_{i}\right)=0, \quad \Phi\left(y, x, v_{i}\right)=0 .
$$

The function $\varphi\left(x, y, u_{i}\right)$ is a polynomial of degree 2 in $y$ and degree 1 in $x$, the polynomial $\Phi\left(y, x, v_{i}\right)$ has degree 2 in $x$ and degree 1 in $y$. We have:

$$
\begin{aligned}
& \varphi\left(x, y_{i}, u_{i}\right)=\left(c\left(y_{i}\right) u_{i}-a\left(y_{i}\right)\right) x+\left(d\left(y_{i}\right) u_{i}-b\left(y_{i}\right)\right) \equiv 0, \\
& \Phi\left(y, x_{i}, v_{i}\right)=\left(C\left(x_{i}\right) v_{i}-A\left(x_{i}\right)\right) y+\left(D\left(x_{i}\right) v_{i}-B\left(x_{i}\right)\right) \equiv 0,
\end{aligned}
$$

therefore $\varphi\left(x, y, u_{i}\right)$ is divisible by $y-y_{i}$, and $\Phi\left(y, x, v_{i}\right)$ is divisible by $x-x_{i}$. Set

$$
\varphi\left(x, y, u_{i}\right)=\left(y-y_{i}\right) \gamma_{i}(x, y), \quad \Phi\left(y, x, v_{i}\right)=\left(x-x_{i}\right) \Gamma_{i}(x, y) .
$$

Obviously, both polynomials $\gamma_{i}(x, y)$ and $\Gamma_{i}(x, y)$ are linear in $x$ and $y$, i.e. equating them to zero defines curves of bidegree $(1,1)$. We claim that the equations

$$
\gamma_{i}(x, y)=0 \quad \text { and } \quad \Gamma_{i}(x, y)=0
$$

are equivalent, so that the curve $\mathcal{C}_{i}$ is given by either of them. This is done by demonstrating that either of these equations defines the curve with the properties listed in the theorem. Since these properties determine the curve uniquely, the claim will follow. This demonstration is performed for each type $\mathcal{T}$ separately. Since the argument are to a large extent analogous, we restrict ourselves to details for two extreme cases $\mathcal{T}=\mathrm{I}$ and $\mathcal{T}=\mathrm{V}$ only.

For $\mathcal{T}=\mathrm{I}$, we have: $\varphi\left(x_{j}, y_{j}, u\right)=\Phi\left(y_{j}, x_{j}, v\right) \equiv 0$ for all $1 \leq j \leq 4$. It follows that $\gamma_{i}\left(x_{j}, y_{j}\right)=\Gamma_{i}\left(x_{j}, y_{j}\right)=0$ for all $j \neq i$.

For $\mathcal{T}=\mathrm{V}$, we have to demonstrate that the following holds:
a) $\gamma_{1}\left(x_{1}, y_{1}\right)=0$
b) $\gamma_{1 x}\left(x_{1}, y_{1}\right) \dot{x}_{1}+\gamma_{1 y}\left(x_{1}, y_{1}\right) \dot{y}_{1}=0$,
c) $2 \gamma_{1 x y}\left(x_{1}, y_{1}\right) \dot{x}_{1} \dot{y}_{1}+\gamma_{1 x}\left(x_{1}, y_{1}\right) \ddot{x}_{1}+\gamma_{1 y}\left(x_{1}, y_{1}\right) \ddot{y}_{1}=0$, 
and the same with the replacement of $\gamma_{1}$ by $\Gamma_{1}$. We do this for $\gamma_{1}$ only, since for $\Gamma_{1}$ everything is similar. Claim a) is proved by the following computation:

$$
\begin{aligned}
\gamma_{1}\left(x_{1}, y_{1}\right) & =\lim _{y \rightarrow y_{1}} \frac{\varphi\left(x_{1}, y, u_{1}\right)}{y-y_{1}}=\varphi_{y}\left(x_{1}, y_{1}, u_{1}\right) \\
& =\left(c^{\prime}\left(y_{1}\right) x_{1}+d^{\prime}\left(y_{1}\right)\right) u_{1}-\left(a^{\prime}\left(y_{1}\right) x_{1}+b^{\prime}\left(y_{1}\right)\right) \\
& =-\lambda_{1}\left(c\left(y_{1}\right) u_{1}-a\left(y_{1}\right)\right)=0 .
\end{aligned}
$$

To check claim b), we proceed as follows:

$$
\begin{aligned}
\gamma_{1 x}\left(x_{1}, y_{1}\right) & =\lim _{y \rightarrow y_{1}} \frac{\varphi_{x}\left(x_{1}, y, u_{1}\right)}{y-y_{1}}=\varphi_{x y}\left(x_{1}, y_{1}, u_{1}\right) \\
& =c^{\prime}\left(y_{1}\right) u_{1}-a^{\prime}\left(y_{1}\right), \\
\gamma_{1 y}\left(x_{1}, y_{1}\right) & =\lim _{y \rightarrow y_{1}} \frac{\left(y-y_{1}\right) \varphi_{y}\left(x_{1}, y, u_{1}\right)-\varphi\left(x_{1}, y, u_{1}\right)}{\left(y-y_{1}\right)^{2}} \\
& =\frac{1}{2} \varphi_{y y}\left(x_{1}, y_{1}, u_{1}\right) \\
& =\frac{1}{2}\left(c^{\prime \prime}\left(y_{1}\right) x_{1}+d^{\prime \prime}\left(y_{1}\right)\right) u_{1}-(1 / 2)\left(a^{\prime \prime}\left(y_{1}\right) x_{1}+b^{\prime \prime}\left(y_{1}\right)\right) \\
& =-\lambda_{1}\left(c^{\prime}\left(y_{1}\right) u_{1}-a^{\prime}\left(y_{1}\right)\right)-\frac{1}{2} \lambda_{1}^{3 / 2} \theta_{1}\left(c\left(y_{1}\right) u_{1}-a\left(y_{1}\right)\right) \\
& =-\lambda_{1}\left(c^{\prime}\left(y_{1}\right) u_{1}-a^{\prime}\left(y_{1}\right)\right) .
\end{aligned}
$$

To prove the claim c), we derive further:

$$
\begin{aligned}
\gamma_{1 x y}\left(x_{1}, y_{1}\right) & =\lim _{y \rightarrow y_{1}} \frac{\left(y-y_{1}\right) \varphi_{x y}\left(x_{1}, y, u_{1}\right)-\varphi_{x}\left(x_{1}, y, u_{1}\right)}{\left(y-y_{1}\right)^{2}} \\
& =\frac{1}{2} \varphi_{x y y}\left(x_{1}, y_{1}, u_{1}\right) \\
& =\frac{1}{2}\left(c^{\prime \prime}\left(y_{1}\right) u_{1}-a^{\prime \prime}\left(y_{1}\right)\right) \\
& =-\frac{1}{2} \lambda_{1}^{1 / 2} \theta_{1}\left(c^{\prime}\left(y_{1}\right) u_{1}-a^{\prime}\left(y_{1}\right)\right)-\frac{1}{2} \varkappa_{1} \lambda_{1}^{-1}\left(c\left(y_{1}\right) u_{1}-a\left(y_{1}\right)\right) \\
& =-\frac{1}{2} \lambda_{1}^{1 / 2} \theta_{1}\left(c^{\prime}\left(y_{1}\right) u_{1}-a^{\prime}\left(y_{1}\right)\right) .
\end{aligned}
$$

Now the claim c) becomes equivalent to:

$$
\lambda_{1}^{1 / 2} \theta_{1} \dot{x}_{1} \dot{y}_{1}=\ddot{x}_{1}-\lambda_{1} \ddot{y}_{1},
$$

which, in turn, is equivalent to the second formula in (42).

\section{Blow-up.}

Consider a quadrirational map $F$ of type $\mathcal{T}=$ I, II, III, IV or V. Then the point $\left(x_{i}, y_{i}\right)(i=1, \ldots, m)$ is blown up to the curve $\mathcal{D}_{i}$ on $\mathbb{C P}^{1} \times \mathbb{C P}^{1}$ defined 
as the curve of bidegree $(1,1)$ satisfying the conditions analogous to those listed in Theorem 21. We want to give a more complete analytic picture of the blow-up process.

Theorem 22. Let $\left(x_{i}, y_{i}\right)$ be a singular point of the map $F$, such that $x_{i}, y_{i}$ are roots of $R(x), r(y)$, respectively, of multiplicity $1 \leq \ell \leq 4$. Let $\mathcal{G}_{i}^{(\ell-1)}=$ $\left(\dot{x}_{i}, \dot{y}_{i}, \ldots, x_{i}^{(\ell-1)}, y_{i}^{(\ell-1)}\right)$ be the corresponding singularity data of the map $F$ at the point $\left(x_{i}, y_{i}\right)$.

- If $(x(t), y(t)) \rightarrow\left(x_{i}, y_{i}\right)$ along a curve with a definite $(\ell-1)$-germ different from $\mathcal{G}_{i}^{(\ell-1)}$, then $F(x(t), y(t)) \rightarrow\left(u_{i}, v_{i}\right)$.

- If $(x(t), y(t)) \rightarrow\left(x_{i}, y_{i}\right)$ along a curve with a definite $\ell$-germ whose $(\ell-1)$-germ coincides with $\mathcal{G}_{i}^{(\ell-1)}$, then the images $F(x(t), y(t))$ have a definite limiting point lying on $\mathcal{D}_{i}$. Different $\ell$-germs correspond to different limiting points on $\mathcal{D}_{i}$.

Proof. Note that $\ell$-germs with a fixed $(\ell-1)$-germ $\mathcal{G}_{i}^{(\ell-1)}$ are characterized by one number from $\mathbb{C P}^{1}$ (e.g., for $\ell=1$ - by the slope $\dot{x}(0) / \dot{y}(0)$, for $\ell=2$ — by the curvature $(\ddot{x}(0) \dot{y}(0)-\dot{x}(0) \ddot{y}(0)) /(\dot{x}(0) \dot{y}(0))^{3 / 2}$, etc.) Therefore the second statement of the theorem shows the isomorphism of $\mathcal{D}_{i}$ with $\mathbb{C P}^{1}$. We will give the details of computations for the proof of the second statement in the simplest case $\ell=1$ only. On a line $\left\{(x, y)=\left(x_{i}+\mu t, y_{i}+\nu t\right)\right\}$ through $\left(x_{i}, y_{i}\right)$ the values of $(u, v)=F(x, y)$ have a well-defined limit as $t \rightarrow 0$ :

$$
u \rightarrow \frac{a\left(y_{i}\right) \mu+\left(a^{\prime}\left(y_{i}\right) x_{i}+b^{\prime}\left(y_{i}\right)\right) \nu}{c\left(y_{i}\right) \mu+\left(c^{\prime}\left(y_{i}\right) x_{i}+d^{\prime}\left(y_{i}\right)\right) \nu}, \quad v \rightarrow \frac{A\left(x_{i}\right) \nu+\left(A^{\prime}\left(x_{i}\right) y_{i}+B^{\prime}\left(x_{i}\right)\right) \mu}{C\left(x_{i}\right) \nu+\left(C^{\prime}\left(x_{i}\right) y_{i}+D^{\prime}\left(x_{i}\right)\right) \mu}
$$

We have for these limit points:

$$
\begin{aligned}
\frac{\mu}{\nu} & =\frac{\left(c^{\prime}\left(y_{i}\right) x_{i}+d^{\prime}\left(y_{i}\right)\right) u-\left(a^{\prime}\left(y_{i}\right) x_{i}+b^{\prime}\left(y_{i}\right)\right)}{-c\left(y_{i}\right) u+a\left(y_{i}\right)} \\
& =\frac{-C\left(x_{i}\right) v+A\left(x_{i}\right)}{\left(C^{\prime}\left(x_{i}\right) y_{i}+D^{\prime}\left(x_{i}\right)\right) v-\left(A^{\prime}\left(x_{i}\right) y_{i}+B\left(x_{i}\right)\right)} .
\end{aligned}
$$

So, it remains to prove the following claim:

- If $x_{i}, y_{i}$ are simple roots of $R(x), r(y)$, respectively, then the equation 
of the curve $\mathcal{D}_{i}$ reads:

$$
\begin{aligned}
\left(c\left(y_{i}\right) u-a\left(y_{i}\right)\right) & \left(C\left(x_{i}\right) v-A\left(x_{i}\right)\right) \\
= & \left(\left(c^{\prime}\left(y_{i}\right) x_{i}+d^{\prime}\left(y_{i}\right)\right) u-\left(a^{\prime}\left(y_{i}\right) x_{i}+b^{\prime}\left(y_{i}\right)\right)\right) \\
& \times\left(\left(C^{\prime}\left(x_{i}\right) y_{i}+D^{\prime}\left(x_{i}\right)\right) v-\left(A^{\prime}\left(x_{i}\right) y_{i}+B\left(x_{i}\right)\right)\right) .
\end{aligned}
$$

According to the proof of Theorem 21, applied with $F^{-1}$ instead of $F$, the equations of $\mathcal{D}_{i}$ are given by

$$
\widehat{\varphi}\left(u, v, x_{i}\right) /\left(v-v_{i}\right)=0 \quad \text { or } \quad \widehat{\Phi}\left(v, u, y_{i}\right) /\left(u-u_{i}\right)=0 \text {. }
$$

To show that these equations are equivalent to (50), recall that, for instance,

$$
\begin{aligned}
\widehat{\Phi}(v, u, y) & =(\widehat{C}(u) y+\widehat{D}(u)) v-(\widehat{A}(u) y+\widehat{B}(u)) \\
& =\frac{1}{r(y)}(q(y, u) v-p(y, v)) \\
& =\frac{(c(y) u-a(y))^{2}}{r(y)}((C(X) y+D(X)) v-(A(X) y+B(X))),
\end{aligned}
$$

where

$$
X=X(y, u)=\frac{d(y) u-b(y)}{-c(y) u+a(y)} .
$$

Recall also that for $y=y_{i}$ we have:

$$
X\left(y_{i}, u\right)=\frac{d\left(y_{i}\right) u-b\left(y_{i}\right)}{-c\left(y_{i}\right) u+a\left(y_{i}\right)} \equiv x_{i} .
$$

Therefore:

$$
\begin{aligned}
& \widehat{\Phi}\left(v, u, y_{i}\right)=\frac{\left(c\left(y_{i}\right) u-a\left(y_{i}\right)\right)^{2}}{r^{\prime}\left(y_{i}\right)}\left(C\left(x_{i}\right) v-A\left(x_{i}\right)\right) \\
& +\frac{\left(c\left(y_{i}\right) u-a\left(y_{i}\right)\right)^{2}}{r^{\prime}\left(y_{i}\right)}\left(\left(C^{\prime}\left(x_{i}\right) y_{i}+D^{\prime}\left(x_{i}\right)\right) v-\left(A^{\prime}\left(x_{i}\right) y_{i}+B\left(x_{i}\right)\right)\right) X_{y}\left(y_{i}, u\right) .
\end{aligned}
$$

It is easy to calculate that:

$$
X_{y}\left(y_{i}, u\right)=\frac{\left(c^{\prime}\left(y_{i}\right) x_{i}+d^{\prime}\left(y_{i}\right)\right) u-\left(a^{\prime}\left(y_{i}\right) x_{i}+b^{\prime}\left(y_{i}\right)\right)}{-c\left(y_{i}\right) u+a\left(y_{i}\right)} .
$$


Noting also that $c\left(y_{i}\right) u-a\left(y_{i}\right)=c\left(y_{i}\right)\left(u-u_{i}\right)$, we find finally the equations of the curve $\mathcal{D}_{i}$ in the required form (50):

$$
\begin{aligned}
\frac{r^{\prime}\left(y_{i}\right)}{c\left(y_{i}\right)} \cdot \frac{\widehat{\Phi}\left(v, u, y_{i}\right)}{u-u_{i}}=\left(c\left(y_{i}\right) u-a\left(y_{i}\right)\right)\left(C\left(x_{i}\right) v-A\left(x_{i}\right)\right) \\
-\left(\left(c^{\prime}\left(y_{i}\right) x_{i}+d^{\prime}\left(y_{i}\right)\right) u-\left(a^{\prime}\left(y_{i}\right) x_{i}+b^{\prime}\left(y_{i}\right)\right)\right) \\
\times\left(\left(C^{\prime}\left(x_{i}\right) y_{i}+D^{\prime}\left(x_{i}\right)\right) v-\left(A^{\prime}\left(x_{i}\right) y_{i}+B\left(x_{i}\right)\right)\right) .
\end{aligned}
$$

This proves the second statement of the theorem for $\ell=1$. Calculation for $\ell>1$ are similar, as well as the proof of the first statement.

\section{Subclasses $[1: 2]$ and $[1: 1]$.}

In this Appendix we discuss briefly maps of the subclasses [1:2] and [1:1] (cf. Sect. 4). According to Lemma 7, these maps are described by the polynomial systems of the form

$$
\Phi(y, x, v)=f(y, v) x+g(y, v)=0, \quad \widehat{\Phi}(y, u, v)=\widehat{f}(y, v) u+\widehat{g}(y, v)=0,
$$

where $f(y, v)=f_{1} y v+f_{2} y+f_{3} v+f_{4}$ and analogously for $g, \widehat{f}, \widehat{g}$. In order to find a canonical form of this system with respect to the transformations from $(\mathcal{M} \ddot{o} b)^{4}$, notice that Möbius transformations $x \rightarrow(\varkappa x+\mu) /(\lambda x+\nu)$ lead to $f \rightarrow \varkappa f+\lambda g, g \rightarrow \mu f+\nu g$. They can be used to turn the polynomials $f, g$ into reducible ones. It is easy to see that this is possible if and only if the characteristic polynomial

$$
\operatorname{det}\left(\varkappa\left(\begin{array}{ll}
f_{1} & f_{2} \\
f_{3} & f_{4}
\end{array}\right)+\lambda\left(\begin{array}{ll}
g_{1} & g_{2} \\
g_{3} & g_{4}
\end{array}\right)\right)
$$

has two distinct eigenvalues $(\varkappa: \lambda),(\mu: \nu) \in \mathbb{C P}^{1}$. If this is true also for the second equation of the system (51) (i.e. if the polynomials $\widehat{f}, \widehat{g}$ can be made reducible by a Möbius transformation in $u$ ), then the map (51) can be brought into the form

$$
\begin{aligned}
& x\left(y-y_{0}\right)\left(v-v_{0}\right)+\left(y-y_{1}\right)\left(v-v_{1}\right)=0, \\
& u\left(y-y_{2}\right)\left(v-v_{2}\right)+\left(y-y_{3}\right)\left(v-v_{3}\right)=0,
\end{aligned}
$$

with some $y_{i}, v_{i} \in \mathbb{C P}^{1}$. If each quadruple $\left(y_{i}\right)$ and $\left(v_{i}\right)$ contains four distinct points then suitable Möbius transformations in $y$ and $v$ bring these quadruples into $(\infty, 0, \beta, 1)$ and $(\alpha, 1, \infty, 0)$, respectively, and the system (52) - into the form

$$
x(v-\alpha)-y(v-1)=0, \quad u(y-\beta)-v(y-1)=0 .
$$


This results in the map

$$
G_{\alpha, \beta}: \quad u=\frac{(\alpha x-y)(y-1)}{(x-y)(y-\beta)}, \quad v=\frac{\alpha x-y}{x-y} .
$$

This is the canonical form of the generic map from the subclass [1:2]. Several degeneracies can happen, due to coincidence of some points $\left(y_{i}\right)$ or $\left(u_{i}\right)$ in (52), as well as the case of double eigenvalue $(\varkappa: \lambda)=(\mu: \nu)$, for one or both equations in (51). We leave the analysis of these degenerate cases to the interested reader.

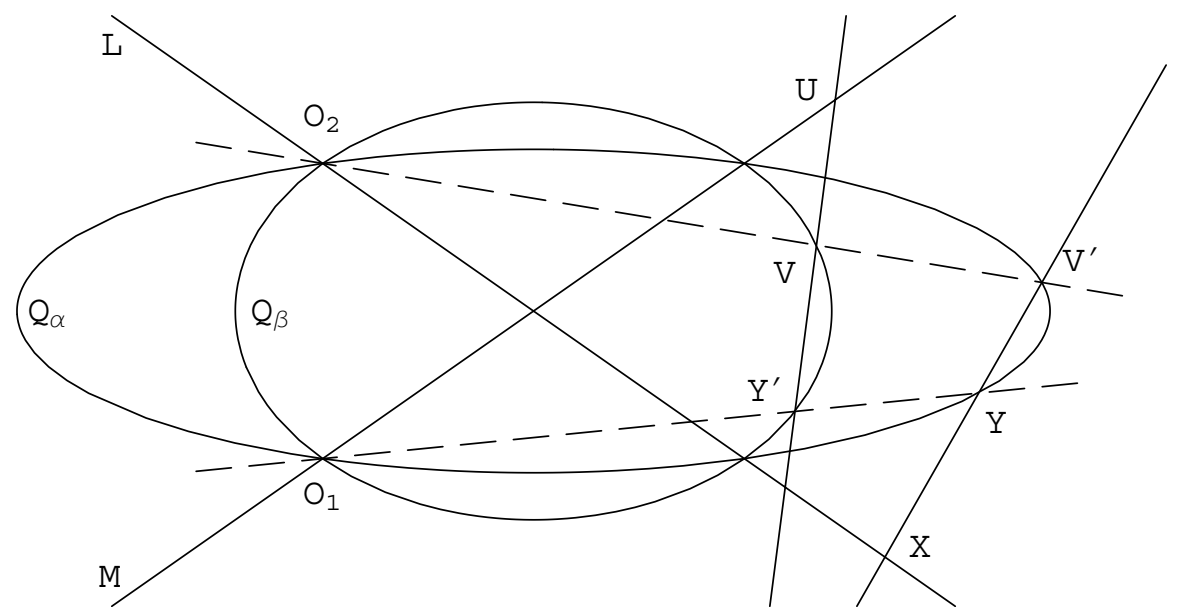

Figure 6: The geometric interpretation of the map from [1:2] subclass

Surprisingly, the geometric interpretation of the map (53) is somewhat more complicated than the one presented on Fig. 4. The construction actually includes three conics from the linear pencil: the pair of nondegenerate conics $Q_{\alpha}, Q_{\beta}$, and a degenerate conic which is a pair of straight lines $L \cup M$. Fix two points $O_{1}, O_{2}$ of the base locus of the pencil (consisting in the present generic case of four points). Choose the degenerate conic $L \cup M$ so that neither of these lines contains both of $O_{i}$. The projections from the points $O_{1}, O_{2}$ establish two different isomorphisms of the nondegenerate conics of the pencil. The geometric map described by the formulas (53) is $\mathcal{F}: L \times Q_{\alpha} \ni(X, Y) \mapsto(U, V) \in M \times Q_{\beta}$ constructed as follows:

- The point $V^{\prime}$ is the intersection point of $Q_{\alpha} \cap \overline{X Y}$ differerent from $Y$, and $V \in Q_{\beta}$ is obtained from $V^{\prime} \in Q_{\alpha}$ via projection from $O_{2}$. 
- The point $Y^{\prime} \in Q_{\beta}$ is obtained from $Y \in Q_{\alpha}$ via projection from $O_{1}$, and $U$ is the intersection of $M$ with $\overline{V Y^{\prime}}$.

This construction is illustrated on Fig. 6 (the two identifying projections are shown by the dashed lines). The map (53) results upon fixing the objects on this figure as follows. Use non-homogeneous coordinates $\left(W_{1}, W_{2}\right)$ on the affine part $\mathbb{C}^{2}$ of $\mathbb{C P}^{2}$. The base locus of the pencil consists of the four points $O_{1}=(0,0), O_{2}=(1,0),(0,1)$ and $(1,1)$. The equations and the parametrizations of the lines and conics are:

$$
\begin{array}{rlrl}
L: & W_{1}+W_{2}=1, & \left(W_{1}, W_{2}\right) & =\left(\frac{1}{1+x}, \frac{x}{1+x}\right), \\
M: & W_{1}=W_{2}, & \left(W_{1}, W_{2}\right) & =\left(\frac{1}{1+u}, \frac{1}{1+u}\right), \\
Q_{\alpha}: & W_{2}\left(W_{2}-1\right)=\alpha W_{1}\left(W_{1}-1\right), & \left(W_{1}, W_{2}\right) & =\left(\frac{y-\alpha}{y^{2}-\alpha}, \frac{y(y-\alpha)}{y^{2}-\alpha}\right) \\
& =\left(\frac{v-\alpha}{v^{2}-\alpha}, \frac{\alpha(v-1)}{v^{2}-\alpha}\right) .
\end{array}
$$

So, the points $X \in L$ and $Y \in Q_{\alpha}$ are parametrized by the slope $x$, resp. $y$, of the line $\overline{O_{1} X}$, resp. $\overline{O_{1} Y}$, while the points $U \in M$ and $V \in Q_{\beta}$ are parametrized by the slope $-u$, resp. $-v$, of the line $\overline{O_{2} U}$, resp. $\overline{O_{2} V}$.

Note that by $\alpha=\beta$ only two conics $\left(Q_{\beta}\right.$ and the degenerate one $L \cup$ $M)$ are involved, and the projections from $O_{1}$ and $O_{2}$ drop out from the construction. Correspondingly, the map (53) with $\alpha=\beta$ may be obtained from the map of Sect. 2 by considering two different parametrizations for $X, Y$ and for $U, V$, as above, and by letting one of the two conics degenerate. More precisely, the map $\left(F_{\mathrm{I}}\right)$ under the Möbius transformations $u \mapsto \alpha(u-$ $1) /(u-\alpha), v \mapsto \beta(v-1) /(v-\beta)$ turns into

$$
\widehat{F}_{\alpha, \beta}: \quad u=\frac{(\beta x-\alpha y)(y-1)}{(x-y)(y-\beta)}, \quad v=\frac{(\beta x-\alpha y)(x-1)}{(x-y)(x-\alpha)},
$$

and setting here $\alpha=1$ (i.e. letting $Q_{\alpha}$ degenerate) leads to the same map as setting $\alpha=\beta$ in (53).

Maps of the subclass [1:2] can be included into 3D-consistent systems, the consistency being governed by matching singularities along the edges of the elementary cube, like in Theorem 19. Not going into details, we provide the reader just with a generic instance of such a construction. Let both facets of the cube on Fig. 3 parallel to the coordinate plane $x y$ carry the $[1: 2]$ map

$$
\widehat{G}_{\alpha, \beta}: \quad u=\frac{(\beta x-\alpha y)(y-1)}{(x-y)(y-\beta)}, \quad v=\frac{(\beta x-\alpha y)}{\alpha(x-y)},
$$


both facets parallel to the coordinate plane $x z$ carry the $[1: 2]$ map $\widehat{G}_{\alpha, \gamma}$, while the facets parallel to the coordinate plane $y z$ carry the $[2: 2]$ maps $\widehat{F}_{\beta, \gamma}$ and $\widehat{F}_{\beta / \alpha, \gamma / \alpha}$ (the left and the right ones, respectively). Then this system of maps is $3 \mathrm{D}$ consistent. (Note that $\widehat{G}_{\alpha, \beta}$ coincides with $G_{\beta / \alpha, \beta}$ from (53) up to a scaling of the variable $u$ ).

Finally, note that the [1:1] maps may be interpreted in the same way, with two degenerate conics (two pairs of lines). A generic map of this subclass is a parameter-free one: $u=(x+y-1) / y, v=(x+y-1) / x$. This map is 3D consistent, so that its companion $u=x v /(1-x+x v), y=1-x+x v$ is a Yang-Baxter map (example given in $[5,13]$ ).

\section{References.}

[1] V.E. Adler. Recutting of polygons. Funct. Anal. Appl. 27:2 (1993) 7980 .

[2] V.E. Adler, A.I. Bobenko, Yu.B. Suris. Classification of integrable equations on quad-graphs. The consistency approach. Comm. Math. Phys., 2003, 233, 513-543.

[3] M. Berger. Geometry. Springer-Verlag, Berlin 1987.

[4] A.I. Bobenko, Yu.B. Suris. Integrable systems on quad-graphs. Int. Math. Res. Notices, 2002, No. 11, 573-611.

[5] V.G. Drinfeld. On some unsolved problems in quantum group theory. Lect. Notes in Math., 1992, 1510, 1-8.

[6] P. Etingof. Geometric crystals and set-theoretical solutions to the quantum Yang-Baxter equation. Commun. Algebra, 2003, 31, 1961-1973.

[7] P. Etingof, T. Schedler, A. Soloviev. Set-theoretical solutions to the quantum Yang-Baxter equation. Duke Math. J., 1999, 100, 169-209.

[8] V.M. Goncharenko, A.P. Veselov. Yang-Baxter maps and matrix solitons. Preprint math-ph/0303032.

[9] J. Hietarinta. Permutation-type solutions to the Yang-Baxter and other n-simplex equations. J. Phys. A: Math. Gen. 30 (1997) 4757-4771.

[10] K. Kajiwara, M. Noumi, Y. Yamada. Discrete dynamical systems with $W\left(A_{m-1}^{(1)} \times A_{m-1}^{(1)}\right)$ symmetry. Lett. Math. Phys., 2002, 60, 211-219. 
[11] J.-H. Lu, M. Yan, Y.-Ch. Zhu. On the set-theoretical Yang-Baxter equation. Duke Math. J., 2000, 104, 1-18.

[12] M. Noumi, Y. Yamada. Affine Weyl groups, discrete dynamical systems and Painlevé equations. Comm. Math. Phys. 199:2 (1998) 281-295.

[13] A. Odesskii. Set-theoretical solutions to the Yang-Baxter relation from factorization of matrix polynomials and $\theta$-functions. Moscow Math. J., 2003, 3, 97-103.

[14] Yu.B. Suris, A.P. Veselov. Lax pairs for Yang-Baxter maps. J. Nonlin. Math. Phys., 2003, 10, Suppl.2, 223-230.

[15] A.P. Veselov. Yang-Baxter maps and integrable dynamics. Phys. Lett. A, 2003, 314, 214-221.

[16] A.P. Veselov. Integrable mappings. Russian Math. Surveys 46:5 (1991) $1-51$. 
